\title{
Non-extremal black hole microstates: fuzzballs of fire or fuzzballs of fuzz?
}

\author{
losif Bena, Andrea Puhm and Bert Vercnocke \\ Institut de Physique Théorique, CEA Saclay, \\ 91191 Gif sur Yvette, France \\ E-mail: iosif.bena@cea.fr, andrea.puhm@cea.fr, bert.vercnocke@cea.fr
}

\begin{abstract}
We construct the first family of microstate geometries of near-extremal black holes, by placing metastable supertube probes inside certain scaling supersymmetric smooth microstate geometries. These fuzzballs differ from the classical black hole solution macroscopically at the horizon scale, and for certain probes the fluctuations between various fuzzballs will be visible as thermal noise far away from the horizon. We discuss whether these fuzzballs appear to infalling observers as fuzzballs of fuzz or as fuzzballs of fire. The existence of these solutions suggests that the singularity of non-extremal black holes is resolved all the way to the outer horizon and this "backwards in time" singularity resolution can shed light on the resolution of spacelike cosmological singularities.
\end{abstract}

Keywords: Black Holes in String Theory, D-branes, Black Holes

ARXIV EPRINT: 1208.3468 


\section{Contents}

1 Introduction 1

2 Supertubes in scaling backgrounds $\quad 5$

2.1 Supertubes in three-charge backgrounds 5

$\begin{array}{lll}2.1 .1 & \text { Supertubes in scaling backgrounds }\end{array}$

$\begin{array}{lll}3 & \text { A seven-center scaling solution } & 7\end{array}$

3.1 Background data 8

3.2 The supertube potential 9

4 Non-extremal microstate throats $\quad 10$

$\begin{array}{lll}4.1 \text { The idea } & 10\end{array}$

$\begin{array}{lll}4.2 & \text { Non-extremal black hole parameters } & 13\end{array}$

$\begin{array}{lll}4.3 & \text { Comparing the microstates and the black hole } & 14\end{array}$

$\begin{array}{ll}4.4 \text { The range of validity of our construction } & 15\end{array}$

$\begin{array}{ll}\text { 4.5 The force on probe branes } & 17\end{array}$

5 Fuzzballs of fire or fuzzballs of fuzz (in lieu of conclusions) 19

5.1 What does an in-falling observer see? 20

5.2 Is Hawking radiation coming from brane-flux annihilation? 22

5.3 Are spacelike singularities resolved backwards in time? 23

A Smooth scaling backgrounds $\quad 24$

A.1 Smoothness and regularity 24

$\begin{array}{ll}\text { A.2 Asymptotic charges and angular momenta } & 26\end{array}$

B Non-extremal black hole geometry 26

$\begin{array}{ll}\text { C Approximation for throat depth } & 27\end{array}$

\section{Introduction}

According to the fuzzball proposal [1-6], black holes are a coarse grained-description of an ensemble of horizonless microstate configurations that have the same mass, charges and angular momenta as the classical black hole, but differ from it at the scale of the horizon. One has by now succeeded to construct very large classes of microstate geometries [7-13] for supersymmetric black holes, as well as for non-supersymmetric extremal black holes [14, $15,15-19]$, and these geometries can be thought of as describing the various channels for the resolution of the timelike singularity inside the horizon of these extremal black holes. 


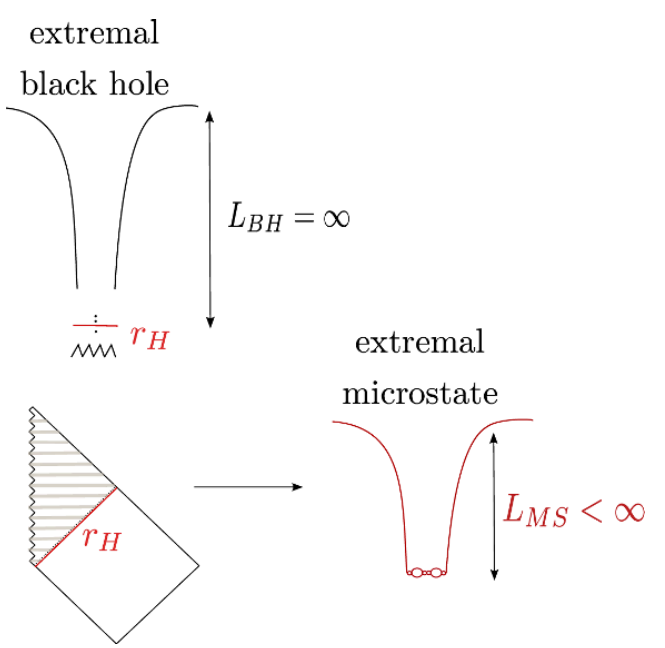

(a) Extremal black holes.
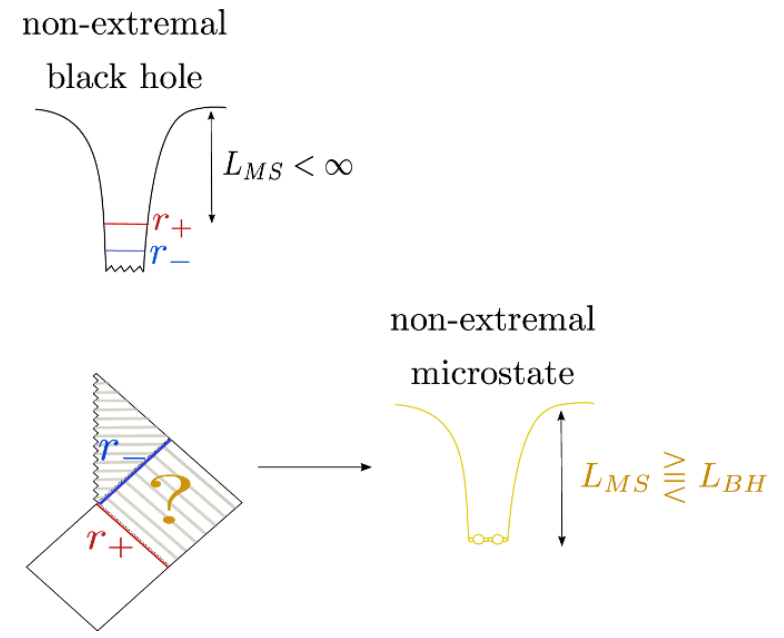

(b) Non-extremal black holes.

Figure 1. Singularity resolution scale.

These resolution channels modify the singular geometry to a large distance away from the singularity, exactly as it happens in other well-understood string-theoretic resolutions of timelike singularities, like Polchinski-Strassler [20], Klebanov-Strassler [21] or LLM [22, 23]. This picture is also supported by analyzing the physics of instabilities [24-28] inside the horizon of extremal black holes.

On the other hand, the scale of the resolution of the singularity of non-extremal black holes is much harder to estimate. The fuzzball proposal and the yearning to solve the black hole information paradox (see [29] for recent work) would have the black hole singularity resolved all the way to the outer horizon, backwards in time from the singularity. The recent "firewall" arguments of $[30,31]$ appear to lead in the same direction. ${ }^{1}$

However, if one is to simply extrapolate the extended evidence for extremal black hole fuzzballs to non-extremal ones, it is well-possible that the timelike singularity of nonextremal black holes is only resolved to the scale of the inner horizon, and that the region between the inner and the outer horizon is still described by the classical black hole solution. This second possibility would not solve the information paradox, but since it does not involves backwards-in-time singularity resolutions it is much easier to the palate than the fuzzball/firewall proposals. An illustration of the two possibilities is given in figure 1 .

To address the question at which scale the singularity resolution happens, one needs to attack the formidable task of constructing non-extremal black hole microstate geometries, which is highly nontrivial. Only two solutions are known: JMaRT [37-39] and the runningBolt [40, 41]; they are very non-generic, and their generalization is nowhere in sight. In [42] we argued for a way to bypass these limitations, and construct instead microstates of nearextremal black holes by adding probes to extremal BPS geometries. We have found that supertubes placed in generic bubbling solutions can have metastable vacua, that can decay

\footnotetext{
${ }^{1}$ For other related works see [32-36].
} 
into the supersymmetric ones by brane-flux annihilation, exactly as it happens when one places antibranes [43] in the Klebanov-Strassler geometry [21].

In this paper we want to take this technology one step further, and to use metastable supertubes to systematically construct microstates of near-extremal black holes. We start from supersymmetric microstate geometries that have the same mass, charges and angular momentum as a supersymmetric three-charge black hole, and have a very long throat (hence they correspond to a scaling solution from the perspective of $4 \mathrm{D}$ supergravity [9, $11,44])$. We construct near-extremal black hole microstate solutions by placing metastable supertubes in these supersymmetric microstate geometries.

There are two ways to obtain such long-throat supersymmetric solutions. The first is to consider a general scaling multicenter solution and tune the length of the throat by moving the centers near each other $[11,44,45]$. The other is to keep the centers aligned on an axis, and to bring them closer and closer by tuning their charges by hand [9]. ${ }^{2}$ The advantage of the second approach is that it produces five-dimensional solutions with $\mathrm{U}(1) \times \mathrm{U}(1)$ invariance, and in these solutions the physics of metastable supertubes is under much better control than in scaling solutions with less symmetry.

As we discussed in [42], the supersymmetry of solutions with metastable supertubes is broken by the relative orientation of the electric charges of the supertube with respect to the solution. Furthermore, we will consider supertubes whose charges are much smaller than those of the background, so we expect generically that their backreaction will give smooth solutions with long throats, that have more mass than charge, and hence are microstates of non-extremal black holes.

Indeed, it was shown in [46] that in the $6 \mathrm{D}$ duality frame where the supertube charges correspond to D1 and D5 branes, a supertube in a bubbling solution backreacts into a smooth supergravity solution. The smoothness is ensured by certain conditions near the supertube, which are identical to those coming from minimizing the DBI probe supertube action; thus we expect that the backreaction of a probe supertube at its minimum, supersymmetric or not, will always give a smooth solution. Another way of seeing this is to recall that the supertube is an object that locally preserves 16 supersymmetries, and all these objects can be dualized into fluxed D6 branes, whose eleven-dimensional uplift is smooth [10]; for metastable supertubes all these supersymmetries are incompatible with those of the background, but this is not something that is visible in the near-supertube region, and hence does not affect the smoothness.

One can worry that the extra supertube charges, though small, may disturb the delicate balance of charges needed to create a long throat. Indeed, in a long throat the leading contributions to the bubble equations cancel, and the supertube contributions may end up being of the same order or larger than the subleading leftovers. However, this is not a problem; even if a supertube changes significantly the length of a throat, one can always tune the flux between cycles by a tiny amount to change this length back to the original one, and this gives a very small correction to the overall charges of the solution.

\footnotetext{
${ }^{2}$ This method has also been used to obtain extremal non-supersymmetric scaling solutions [19].
} 
Having obtained large classes of microstates of near-extremal black holes, we can go on and attack the much harder problem of figuring out what is the physics of these microstates. Indeed, according to the fuzzball proposal one expects the microstates to have the same size as the black hole, but there are various scenarios of how this can happen. It is possible that microstates extend only microscopically away from the horizon, and hence, as suggested in [30], they could give nothing more than a realization of a stretched horizon. Alternatively, the fuzzballs can differ from the black hole on a scale comparable to that of the horizon, and hence give very different physics.

These questions do not make sense for BPS and extremal black holes, and cannot be answered using the BPS black hole microstates constructed so far. Indeed, the thickness of BPS throats is completely determined by the charges, and hence a fuzzball and a black hole that have the same charges automatically have throats of equal thickness. Furthermore, the length of the throat of the black hole is infinite, while the length of the throat of the fuzzballs is always very large but finite, ${ }^{3}$ which does not allow for a meaningful size comparison. On the other hand, near-extremal black holes have throats of finite length, which one can compare with the throat lengths of the family of fuzzballs we construct. The thicknesses of the throats are still automatically equal, because they are mostly controlled by the charges in the near-extremal limit.

As we will see, we are able to construct microstates whose throat has the same length as that of a non-extremal black hole, but we can also obtain with equal ease microstate geometries that have the same mass and charges as the non-extremal black hole, but whose throats are longer or shorter. As far as our construction is concerned, there appears to be no dynamical reason why throats of the same length as the black hole are preferred over longer or shorter ones, and this indicates that the fuzzballs of non-extremal black holes will not differ from the black hole only at microscopic distances from the horizon. ${ }^{4}$

The fuzzball geometries we construct can be used to extract other pieces of physics that have been inaccessible until now. For example, one can use a KKLMMT-type argument [49] to find the forces with which our fuzzballs attract various D-branes, and compare these forces to those of the corresponding black hole. One can also compute the tunneling probabilities of the metastable supertube to the supersymmetric minimum, and compare this to the Hawking radiation rate of the near-extremal black hole; we leave this for future work.

This paper is organized as follows. In section 2 we recall the Hamiltonian of supertubes in three-charge backgrounds. We then focus on a scaling solution with seven centers in section 3 and plot the potential of a typical probe supertube in this background. In section 4 we discuss the interpretation of our configurations as microstates (or fuzzballs) of non-extremal black holes, and compare their properties to those of black holes. In section 5 we discuss whether the fuzzballs we construct appear as fuzzballs of fuzz or as fuzzballs of fire to incoming observers, we speculate on the implication of this work for

\footnotetext{
${ }^{3}$ The only way to figure out whether a long BPS microstate is typical is to compare its length to the mass gap of the typical microstate in the dual CFT, and this comparison indicates that long microstates whose angular momentum is of order one belong indeed the sector where the typical microstates live [9, 11, 47].

${ }^{4}$ Most likely there will be a distribution of fuzzballs of various lengths, and the length of the typical ones will come out to be same as the length of a black hole by some entropy enhancement reason [48].
} 
our understanding of the resolution of spacelike singularities in String Theory, and discuss possible future directions. In appendix A we review the construction and the physics of smooth BPS black hole microstate solutions, in appendix B, we recall the geometry of the non-extremal black hole, and in appendix $\mathrm{C}$ we present an approximation that allows us to compare very easily the lengths of a black-hole throat and of a fuzzball throat.

\section{Supertubes in scaling backgrounds}

In this section, we review the potential for supertubes in a supersymmetric three-charge background. We focus on deep supersymmetric microstate geometries (smooth, horizonless three-charge solutions with scaling behavior) and explain how the scaling effects the supertube potential.

\subsection{Supertubes in three-charge backgrounds}

Consider a supersymmetric background geometry with three charges and three dipole charges, of the type that describes black holes, black rings and their microstate geometries. The metric in the M-theory duality frame in which the three charges correspond to M2 branes wrapping orthogonal $T^{2}$ 's inside $T^{6}$ is $[50,51]$ :

$$
\begin{aligned}
d s_{11}^{2} & =-\left(Z_{1} Z_{2} Z_{3}\right)^{-2 / 3}(d t+k)^{2}+\left(Z_{1} Z_{2} Z_{3}\right)^{1 / 3} d s_{4}^{2}+\left(Z_{1} Z_{2} Z_{3}\right)^{1 / 3} \sum_{I=1}^{3} \frac{d s_{I}^{2}}{Z_{I}} \\
F_{4} & =\sum_{I=1}^{3} d A^{(I)} \wedge \omega_{I}, \quad d A^{(I)}=-d\left[Z_{I}^{-1}(d t+k)\right]+\Theta^{(I)} .
\end{aligned}
$$

where $d s_{I}^{2}$ and $\omega_{I}$ are unit metrics and volume forms on the three orthogonal $T^{2}$ 's and $d s_{4}^{2}$ is the metric of a hyper-Kähler base space. Supersymmetry requires the two-forms $\Theta^{(I)}$ to be self-dual on the base. When the hyper-Kähler space is Gibbons-Hawking (GH) or Taub-NUT:

$$
d s_{4}^{2}=V^{-1}(d \psi+A)^{2}+V d s_{3}^{2} \quad \text { with } \quad d A=\star_{3} d V,
$$

where $d s_{3}^{2}$ is the flat metric on $\mathbb{R}^{3}$, the solution is completely determined by specifying 8 harmonic functions $V, K^{I}, L_{I}, M$ in the $\mathrm{GH}$ base $[52,53]$. The harmonic functions can have sources on an arbitrary number of positions in $\mathbb{R}^{3}$. The warp factors and rotation one-form are given by

$$
\begin{aligned}
Z_{I} & =L_{I}+\frac{1}{2} C_{I J K} V^{-1} K^{J} K^{K}, \\
k & =\mu(d \psi+A)+\omega,
\end{aligned}
$$

with $C_{I J K}=\left|\epsilon_{I J K}\right|$ and

$$
\begin{aligned}
\mu & =\frac{1}{6} C_{I J K} V^{-2} K^{I} K^{J} K^{K}+\frac{1}{2} L_{I} K^{I}+M, \\
\vec{\nabla} \times \vec{\omega} & =V \vec{\nabla} M-M \vec{\nabla} V+\frac{1}{2}\left(K^{I} \vec{\nabla} L_{I}-L_{I} \vec{\nabla} K^{I}\right) .
\end{aligned}
$$


Note that the inverse of the warp factors $Z_{I}$ are also the electric potentials for the four-form and hence they determine the M2 charges at each background center.

In [42] we found the Hamiltonian of a two-charge supertube in such a multicenter three-charge background with a Gibbons-Hawking base. The two charges $q_{1}$ and $q_{2}$ of the supertube are parallel to those of the background and correspond to M2 branes along the first and second $T^{2}$. The dipole charge, $d_{3}$, corresponds to an M5 brane extended along those two tori wrapping the fiber of the Gibbons-Hawking space. The Hamiltonian is:

$$
\begin{aligned}
\mathcal{H}= & \frac{\sqrt{Z_{1} Z_{2} Z_{3} / V}}{d_{3} R^{2}} \sqrt{\left(\tilde{q}_{1}^{2}+d_{3}^{2} \frac{R^{2}}{Z_{2}^{2}}\right)\left(\tilde{q}_{2}^{2}+d_{3}^{2} \frac{R^{2}}{Z_{1}^{2}}\right)}+\frac{\mu V^{2}}{d_{3} R^{2}} \tilde{q}_{1} \tilde{q}_{2}-\frac{1}{Z_{1}} \tilde{q}_{1}-\frac{1}{Z_{2}} \tilde{q}_{2}-\frac{d_{3} \mu}{Z_{1} Z_{2}} \\
& +q_{1}+q_{2},
\end{aligned}
$$

where we have introduced

$$
\tilde{q}_{1} \equiv q_{1}+d_{3}\left(K^{2} / V-\mu / Z_{2}\right), \quad \tilde{q}_{2} \equiv q_{2}+d_{3}\left(K^{1} / V-\mu / Z_{1}\right),
$$

and $R$ is proportional to the size of the Gibbons-Hawking fiber

$$
R^{2} \equiv Z_{1} Z_{2} Z_{3} / V-\mu^{2}
$$

The harmonic functions $K^{1}$ and $K^{2}$ encode two of the three dipole moments of the background. The minima of the potential determine the position on the GH base of (meta)stable supertubes in a given three-charge background. Depending on the relative orientation of the M2 charges of supertube and the background, the minima of the potential will be supersymmetric (with energy $V_{B P S}=q_{1}+q_{2}$ ) or non-supersymmetric.

\subsubsection{Supertubes in scaling backgrounds}

A scaling background is a bubbling configuration that has a set of GH points that can approach each other arbitrarily close, see figure 2 for a cartoon. As the points get closer together, the solution develops an ever deeper throat and looks more and more like the black hole with the same asymptotic charges. See appendix A. Deep scaling solutions are dual to states that belong to the same CFT sector as the typical microstates, that give the leading contribution to the black hole entropy [9, 11, 47].

For a given set of charges, a scaling limit exists if we can find a solution to the bubble equations (A.7) or (A.8) for

$$
r_{i j}=\epsilon \tilde{r}_{i j} \quad \text { with } \quad \epsilon \rightarrow 0 .
$$

When such a solution can be found, all distances in the GH base scale to zero, but the physical size of the bubbles and ratios between distances are preserved throughout the scaling $\epsilon \rightarrow 0$, because the warp factors along the bubbles diverge appropriately.

When we focus on scaling backgrounds we find that the Hamiltonian has a similar scaling. By rescaling the coordinates on the $3 \mathrm{~d}$ base as $\vec{r}=\epsilon \overrightarrow{\vec{r}}$, and taking the limit $\epsilon \rightarrow 0$, the Hamiltonian scales as

$$
\mathcal{H}(\epsilon \overrightarrow{\tilde{r}})=\epsilon \mathcal{H}(\overrightarrow{\tilde{r}})+\mathcal{O}\left(\epsilon^{2}\right)
$$




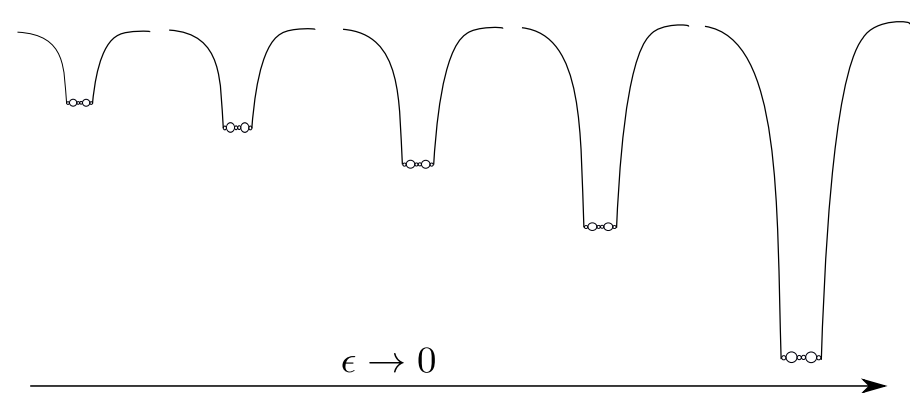

Figure 2. Heuristic picture of scaling microstate geometries.

As mentioned in the Introduction, there exist two ways of obtaining a scaling solution. The first is to consider a set of $N$ centers whose charges allow for scaling behavior; they satisfy $N-1$ bubble equations, and their $2 N-2$ dimensional moduli has a region where the points come together, and the fully backreacted solution develops a long throat $[11,44]$. The second way is to insist that the centers be collinear - their positions are now parameterized by $N-1$ variables that are completely determined by the $N-1$ bubble equations - and force the centers to scale by tuning by hand some of the flux parameters on the centers or some of the moduli of the solution [9].

We will use the second approach, essentially because it gives much more control on the dynamics of the supertube. If one adds a supertube to a scaling solution whose centers are not collinear, the energy of the supertube depends on the length of the throat, and can change as the centers move in the moduli space. On the other hand, in a $\mathrm{U}(1) \times \mathrm{U}(1)$ invariant solution the centers are collinear and hence frozen, and if the supertube charges are smaller than those of the other centers, the physics of the metastable supertube is expected to be captured by its probe action in the background.

Thus, in the examples in the next section, we focus on scaling solutions where all the GH points are collinear and we will 'turn the knob' of the scaling control parameter $\epsilon$ by tuning one of the charges $k_{i}^{I}$.

\section{A seven-center scaling solution}

In this section, we analyze the minima the probe supertubes in a "pincer" supersymmetric scaling background (inspired from [9]) whose centers are colinear in $\mathbb{R}^{3}$ and have $J_{L}=0$. This pincer solution contains a central 'blob' of total GH charge one, as well as two symmetric satellite blobs of GH charge zero. For computational ease, we take a configuration that is made up out of a total of seven points on the GH base: a central blob made from three points, of GH charges $-n, 2 n+1,-n$, and two satellites with two points that have GH charges $-Q$ and $+Q$. The configuration, depicted in figure 3 , is $\mathbb{Z}_{2}$ symmetric, and hence has $J_{L}=0$ by construction.

One can then choose fluxes between the various GH centers such that the total configuration has the charges of a BPS black hole with a macroscopically-large horizon area. The particular choice of fluxes that ensures that a five-point solution has no CTC's was obtained 


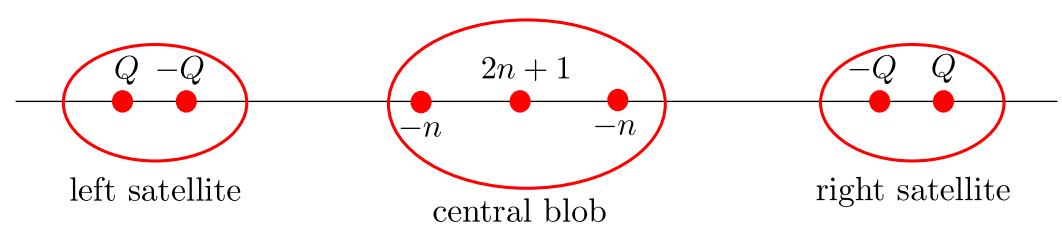

Figure 3. Our setup consists of a central blob of three centers and two satellite blobs of two centers each, with the GH charges as given in the figure.

in [9] by tediously analyzing blob mergers, and our choice is simply the $\mathbb{Z}_{2}$ symmetrization of that choice.

\subsection{Background data}

We choose a cylindrical coordinate system $(\rho, z, \theta)$ in three dimensions, where $z$ runs along the axis through the centers and $\rho, \theta$ are polar coordinates in the orthogonal plane. Since we have cylindrical symmetry, the solution only depends on the coordinates $z$ and $\rho$. The seven centers are put on the $z$-axis and are numbered $z_{1} \ldots z_{7}$ as in figure 4 . We choose the GH charges to be

$$
v_{1}=20, v_{2}=-20, \quad v_{3}=-12, v_{4}=25, v_{5}=-12, \quad v_{6}=-20, v_{7}=20 .
$$

The flux parameters of the central blob are chosen as

$$
k_{i}^{1}=\frac{5}{2}\left|v_{i}\right|, \quad k_{i}^{2}=\hat{k}\left|v_{i}\right|, \quad k_{i}^{3}=\frac{1}{3}\left|v_{i}\right|, \quad i=3,4,5,
$$

and those of the satellites are

$$
\begin{array}{lll}
k_{1}^{1}=1375, & k_{1}^{2}=-\frac{1835}{2}+980 \hat{k}, & k_{1}^{3}=-\frac{8360}{3}, \\
k_{2}^{1}=-1325, & k_{2}^{2}=\frac{1965}{2}-980 \hat{k}, & k_{2}^{3}=\frac{8380}{3},
\end{array}
$$

and their mirror image $k_{7}^{I}=k_{1}^{I}, k_{6}^{I}=k_{2}^{I}$.

The charges of the harmonic functions are then a function of $\hat{k}$ only. For every value of $\hat{k}$, the bubble equations (A.8) fix the position of the seven centers. One can approximate the size of the microstate by $z_{6} \approx r_{0}$ as in [9]:

$$
r_{0}=\frac{\widehat{J}_{L}}{8 \sum_{I}\left(k_{6}^{I}+k_{7}^{I}\right)},
$$

where $\widehat{J}_{L}$ is the angular momentum contained in the centers $z_{3}, \ldots z_{7}$. In the given background, this is linear in $\hat{k}$ as:

$$
r_{0}=\frac{56}{31} \times 10^{3}\left|\hat{k}-\hat{k}_{\star}\right|, \quad k_{\star} \approx 3.17975 .
$$

We will tune $\hat{k}$ such that $r_{0} \rightarrow 0$ and the configuration scales down into a deep throat.

In table 1 we list the relevant distances and ratios of distances for various values of the flux parameter $\hat{k}$. Starting from one set of inter-center distances the bubble equations (A.8) successively determine the equilibrium distance for every value of $\hat{k}$ during the scaling. 


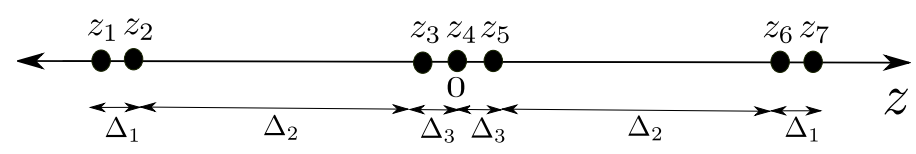

Figure 4. Schematic picture of our microstate configuration.

\begin{tabular}{|c|c|c|c|c|c|}
\hline Background & $\hat{k}$ & $z_{6}$ & $\frac{z_{6}}{r_{0}}$ & $\frac{\Delta_{3}}{\Delta_{1}}$ & $\frac{\Delta_{2}}{\Delta_{1}}$ \\
\hline \hline 1 & 3.08333 & 176.088 & 1.011 & 1.5464 & 6730.9 \\
\hline 2 & 3.16667 & 23.91 & 1.01166 & 1.61449 & 6664.58 \\
\hline 3 & 3.175 & 8.69039 & 1.01279 & 1.6215 & 6657.94 \\
\hline 4 & 3.1775 & 4.12444 & 1.01474 & 1.62362 & 6655.95 \\
\hline 5 & 3.178 & 3.21125 & 1.0158 & 1.62404 & 6655.55 \\
\hline 6 & 3.17833 & 2.60246 & 1.01693 & 1.62432 & 6655.29 \\
\hline 7 & 3.17867 & 1.99366 & 1.01874 & 1.6246 & 6655.02 \\
\hline 8 & 3.1795 & 0.471667 & 1.04441 & 1.62531 & 6654.36 \\
\hline 9 & 3.17967 & 0.167268 & 1.11114 & 1.62545 & 6654.22 \\
\hline
\end{tabular}

Table 1. Distances between the points throughout the scaling process. The distances $\Delta_{i}$ and $z_{6}$ are as in figure 4 . The parameter $\hat{k}$ is tuned for the scaling, all the other charges are kept fixed at their values (3.1) and (3.2). It is clear that the relative distances stay approximately the same during the scaling. Also the total charges $Q_{I}$ and angular momentum $J_{R}$ stay approximately the same throughout the scaling.

Charges and angular momenta. The values of the electric charges and the rightmoving angular momentum as defined in (A.10) and (A.12) stay approximately constant throughout the scaling

$$
Q_{1} \approx 1.476 \times 10^{5}, \quad Q_{2}=1.196 \times 10^{5}, \quad Q_{3} \approx 1.76 \times 10^{5}, \quad \text { and } J_{R} \approx 1.018 \times 10^{8} .
$$

Note that $Q_{2}$ is independent of $\hat{k}$. Since the configuration is symmetric (the charges of opposite centers are the same), the left-moving angular momentum is exactly zero throughout the whole merger process. ${ }^{5}$ Since the charges and angular momenta all stay nearly constant throughout the scaling these microstates have the charges of a black hole of nonzero entropy in all regimes: when they are shallow (before the scaling), when they are very deep (in the scaling limit) and in the whole intermediate regime.

\subsection{The supertube potential}

We plot the potential for a probe supertube in this background, with supertube charges

$$
\left(q_{1}, q_{2}, d_{3}\right)=(10,-50,1) .
$$

\footnotetext{
${ }^{5}$ For configurations with only one satellite this symmetry is broken and $J_{L} \neq 0$. Then $J_{L}$ goes to zero as the solution gets deeper and deeper. The end-point of such a merger is a BMPV black hole microstate with $J_{L}=0$. Only in this deep-throat limit the microstates have the charges of a black hole of non-zero entropy, while our background has the charges of a BMPV black hole throughout the scaling.
} 
The potential is normalized to zero for a supersymmetric minimum:

$$
\tilde{\mathcal{H}} \equiv \mathcal{H}-\left(q_{1}+q_{2}\right)
$$

and we will omit the tilde in the following. For illustrative purposes, we plot the potential of the supertube in the background ' 2 ' of table 1 as a function of $z$ and perform a Contour plot around the minima in a plane through the $z$-axis, see figure 5 . The positions of the seven centers in background 2 are

$$
z_{1}=-23.9136, z_{2}=-23.91, z_{3}=-0.00579078, z_{4}=0, z_{5}=\left|z_{3}\right|, z_{6}=\left|z_{2}\right|, z_{7}=\left|z_{1}\right| .
$$

The potential has several supersymmetric minima: two lie inside the central blob, two lie just outside and there are two more minima in between the central blob and the satellite centers at $z \approx \pm 10$. There are two metastable minima close to the satellites, near $z_{2}$ and $z_{6}$. Since the setup is symmetric, we focus on the metastable minimum at $z_{\mathrm{ms}} \lesssim z_{6}$ :

$$
z_{\mathrm{ms}}=23.8729, \quad z_{6}=23.91 .
$$

The supertube in that minimum can tunnel to the supersymmetric state at $z \simeq 10$ via brane-flux annihilation as explained in [42]. Note that the additional non-supersymmetric minima near $z= \pm 50$ as seen from figure 5 are in fact saddle points and they have a runaway behavior off the axis.

In order to stay well in the probe approximation one needs to make sure that the charges of the supertube are small compared to the charges of the background (as measured by the poles of $L_{I}$ ). In particular, the metastable minimum at $z_{\mathrm{ms}}$ sits close to the centers $z_{6}$ and $z_{7}$ of the background, and we have to make sure that the charges at that position are large compared to the ones of the supertube. For the charges and flux parameters as fixed in (3.1) and (3.2) the background electric charges at the black ring centers are of the order $3 \times 10^{5}$ and hence our supertube is well in the probe regime.

As explained before, the supertube potential scales down linearly with the coordinate distance between the background centers, see eq. (2.11). As an illustration, we compare the potential for two scaling backgrounds, 2 and 9 of table 1 in figure 6 . One clearly sees the self-similarity of the potentials. Also the supertube position $z_{\mathrm{ms}}$ scales down with the throat: its relative position to the other centers stays unchanged.

\section{Non-extremal microstate throats}

Upon backreaction, metastable supertubes in scaling backgrounds should become microstates of a non-extremal black hole. In this section we want to compare the size of these microstates to the size of the corresponding black hole, and understand the scale at which non-extremal fuzzballs differ from the black hole.

\subsection{The idea}

One can estimate the depth of a black hole or of a fuzzball throat by integrating the radial metric component:

$$
L=\int_{r_{\text {bottom }}}^{r_{\text {neck }}} \sqrt{g_{r r}} d r
$$




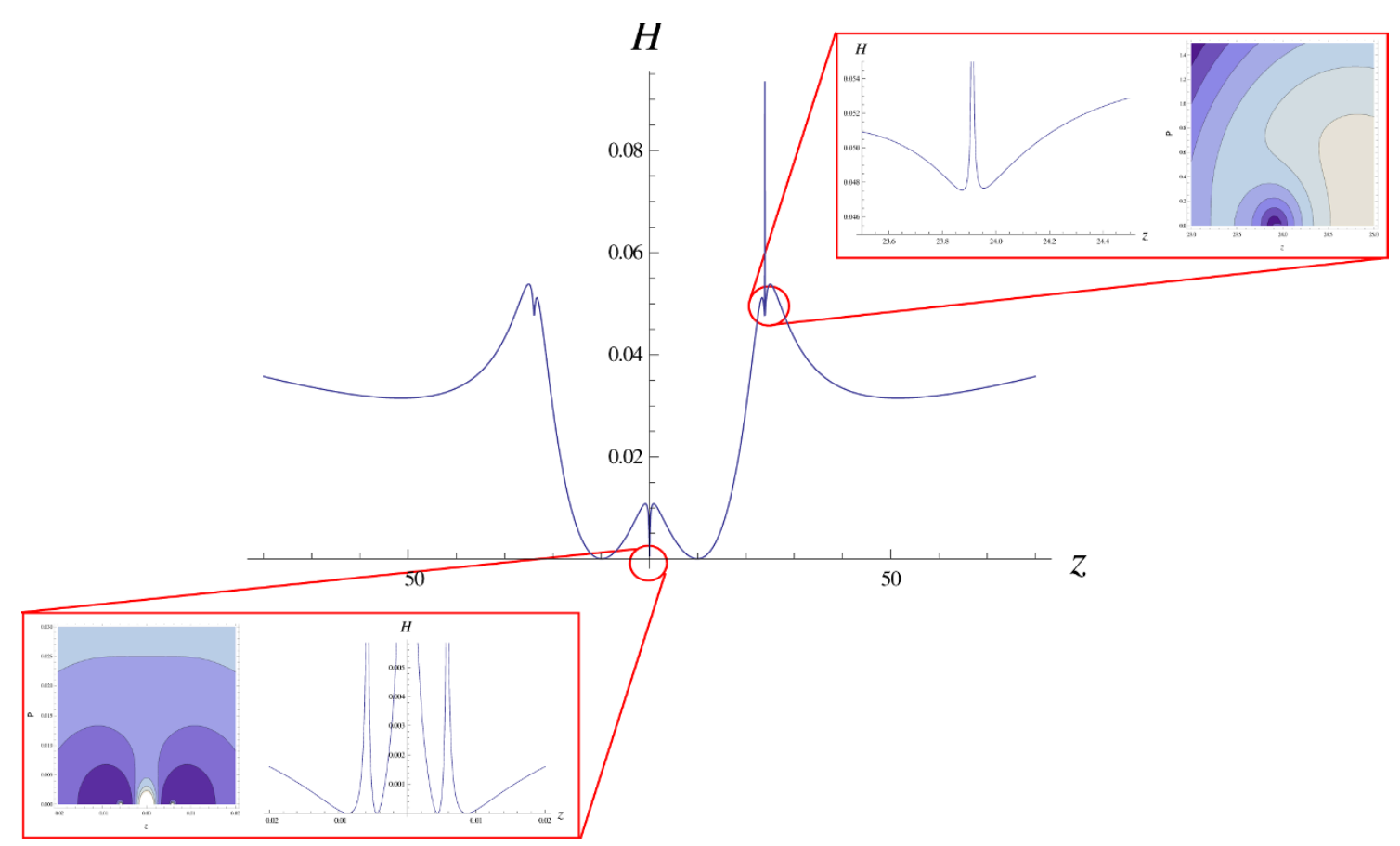

Figure 5. Zoom on the supertube potential for charges $\left(q_{1}, q_{2}, d_{3}\right)=(10,-50,1)$ in background 2. Note the metastable minimum near $z_{6}=23.91$ (and its mirror near $z_{2}=-23.91$ ). The contour plot shows that this minimum is of "Mexican hat - type" in the $z-\rho$ plane around the center $z_{6}\left(z_{2}\right)$; darker colors mean lower energy. On can see that this minimum has no runaway behavior in the $\rho$ direction and hence is truly metastable. The supertube in that minimum can tunnel to a supersymmetric state. Note also that the minima near the central blob are in fact two mirror copies of a Mexican hat-type circular band of minima, as the contour plot in the bottom left corner shows.

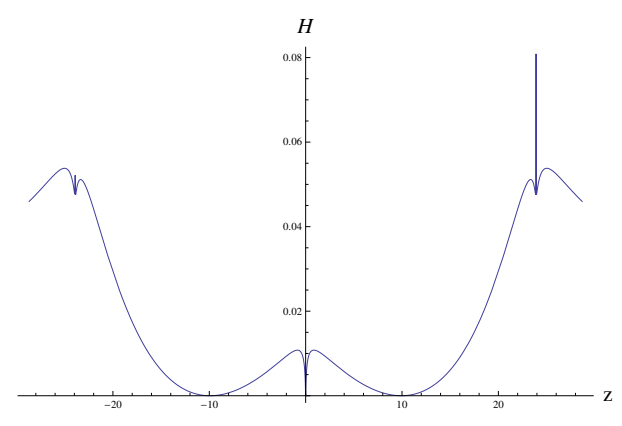

(a) Potential in background $2, z_{6}=23.91$

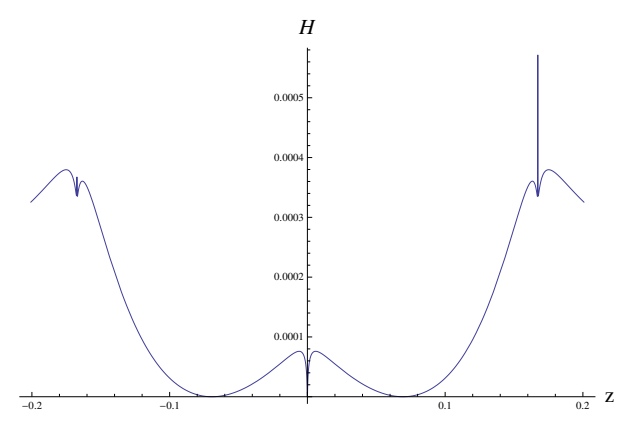

(b) Potential in background $9, z_{6}=$ 0.167268

Figure 6. The supertube potential for charges $\left(q_{1}, q_{2}, d_{3}\right)=(10,-50,1)$ in two scaling backgrounds. The energy scales down linearly with the coordinate size between the centers. 


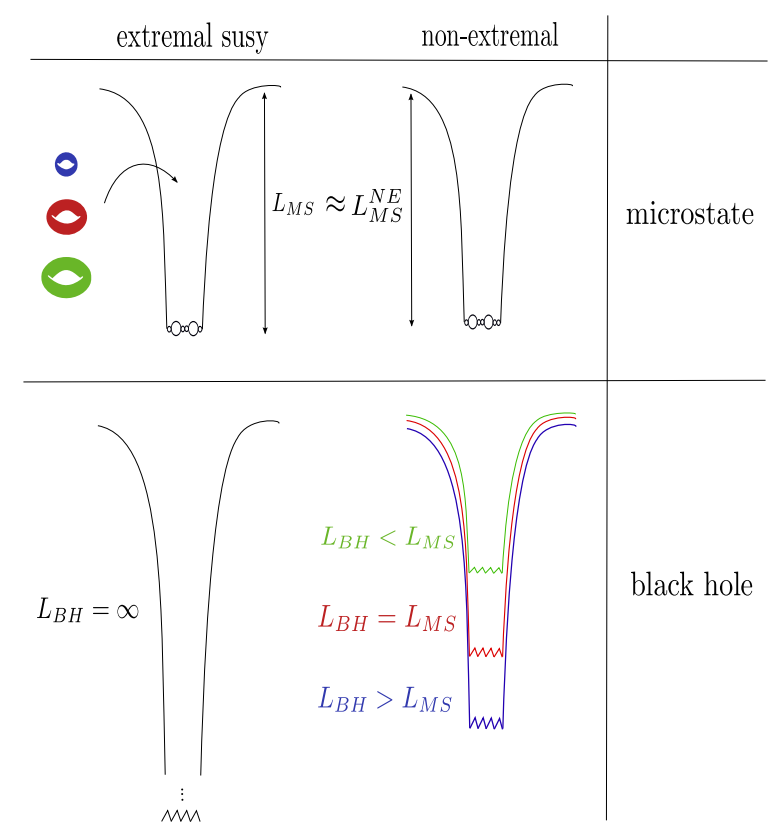

Figure 7. The scaling of the background determines the size of the metastable black hole microstates. For a BPS background of a fixed depth, adding heavy supertubes gives a microstate of a black hole that has shorter throat, while adding light supertubes gives a microstate of a black hole with a longer throat.

between the bottom and the neck of the throat. To get the depth of the non-extremal microstate, we can evaluate this integral in the supersymmetric background geometry since the probe supertube will not affect the geometry too much. We then compare this to the depth of the throat of the non-extremal black hole (a Cvetic-Youm black hole [54], see appendix B) that has the same charges.

The main result of this paper is that we, indeed, find microstates that are of the same depth as the non-extremal black hole, but we also find deeper ones and shallower ones. This is not surprising: Supertube probes placed in deep scaling solutions will not affect the background geometry too much upon backreaction and the resulting non-extremal microstate will, hence, be of the same size as the supersymmetric background. The size of the corresponding non-extremal black hole, however, depends on the extremality parameter which is set by the charges of the supertube. Small supertube charges correspond to deep black holes; increasing the tube charges takes the black hole further away from extremality and thus makes the throat more shallow. Hence, by tuning the supertube charges we can always find the throat of the non-extremal black hole to be of a size comparable to that of its microstates. This intuition is summarized in Figure 7.

In the remainder of this section we make this intuitive picture more precise. First, we determine the data of the non-extremal black hole with the charges of the metastable bound states in section 4.2. We give the depths of the black hole and microstate throats in section 4.3. Since the resulting integrals are quite complicated, we make an insightful approximation in appendix $\mathrm{C}$. 


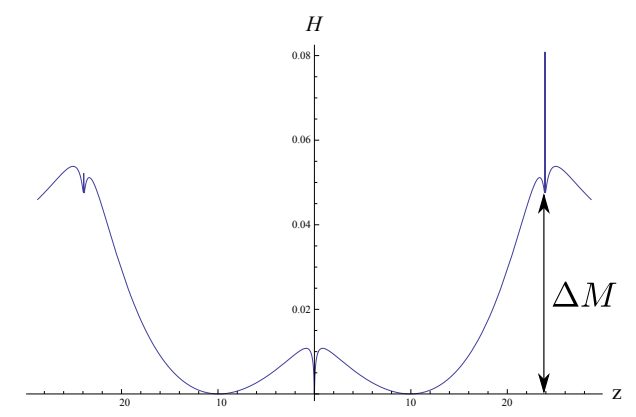

Figure 8. The metastable supertube brings in an excess energy $\Delta M=M_{A D M}^{\mathrm{BH}}-\sum_{I} Q_{I}^{\mathrm{BH}}$.

\subsection{Non-extremal black hole parameters}

We begin with a supersymmetric fuzzball solution that has the charges of a supersymmetric rotating BMPV black hole, and its mass is hence

$$
M=Q_{1}+Q_{2}+Q_{3} .
$$

Adding a supertube with charges $q_{1}, q_{2}$ increases the mass by the value of the supertube potential at the minimum $\mathcal{H}_{\text {min }}=q_{1}+q_{2}+\Delta M$ (see also figure 8 ). When the minimum is supersymmetric $\Delta M=0$, and the resulting configuration is a BPS microstate. When the supertube minimum is metastable, the mass is:

$$
M=Q_{1}+Q_{2}+Q_{3}+q_{1}+q_{2}+\Delta M,
$$

and the charges are

$$
Q_{1}^{\text {tot }}=Q_{1}+q_{1}, \quad Q_{2}^{\text {tot }}=Q_{2}+q_{2} \quad Q_{3}^{\text {tot }}=Q_{3} .
$$

Since, $M_{A D M}>\sum_{I} Q_{I}$, the configuration with a metastable supertube has the charges and mass of a non-extremal black hole. The energy above extremality is exactly given by $\Delta M:$

$$
\Delta M=M-\sum_{I} Q_{I}^{\mathrm{tot}}
$$

In appendix B, we review the non-extremal rotating M2-M2-M2 black hole geometry. The solution depends on six parameters: a mass parameter $m$, three 'boosts' $\delta_{I}$ and angular momentum parameters $a_{1}, a_{2}$ which are related to the ADM mass, charges and angular momenta

$$
\begin{aligned}
M_{A D M}^{\mathrm{BH}} & =\sum_{I} \frac{m}{4}\left(e^{2 \delta_{I}}+e^{-2 \delta_{I}}\right), & J_{1}^{\mathrm{BH}} & =m\left(a_{1} c_{1} c_{2} c_{3}-a_{2} s_{1} s_{2} s_{3}\right), \\
Q_{I}^{\mathrm{BH}} & =\frac{m}{4}\left(e^{2 \delta_{I}}-e^{-2 \delta_{I}}\right), & J_{2}^{\mathrm{BH}} & =-m\left(a_{2} c_{1} c_{2} c_{3}-a_{1} s_{1} s_{2} s_{3}\right),
\end{aligned}
$$

where $c_{I}=\cosh \delta_{I}$ and $s_{I}=\sinh \delta_{I}$. We determine the parameters $\delta_{I}, a_{i}, m$ of the metastable state. The parameters $\delta_{I}$ are given by the charges and parameter $m$ as

$$
\frac{m}{2} e^{2 \delta_{I}}=Q_{I}^{\mathrm{BH}}+\sqrt{\left(Q_{I}^{\mathrm{BH}}\right)^{2}+\frac{m^{2}}{4}} .
$$


The parameter $m$ is determined by the energy of the metastable supertube as follows. With (4.6) the energy above extremality (4.5) can be written in terms of $m$ and $\delta_{I}$ as

$$
\Delta M=\sum_{I} \frac{m}{2} e^{-2 \delta_{I}}
$$

In the probe approximation, the supertube charges are small compared to those of the background. Then the non-extremal black hole is close to the supersymmetric limit $\left(m / Q_{I}^{\mathrm{BH}} \ll 1\right)$ and the black hole charges are approximately those of the background and (4.7) becomes

$$
\frac{m}{4} e^{2 \delta_{I}}=Q_{I}
$$

The non-extremality parameter is then given by the charges and energy of the metastable state

$$
m=\sqrt{\frac{8 \Delta M}{\sum_{I} 1 / Q_{I}}} .
$$

In this approximation, the angular momentum parameters $a_{1}, a_{2}$ are:

$$
\begin{aligned}
& J_{L}^{\mathrm{BH}} \equiv J_{1}^{\mathrm{BH}}-J_{2}^{\mathrm{BH}}=\frac{\sqrt{m}}{2}\left(a_{1}+a_{2}\right) \sqrt{Q_{1} Q_{2} Q_{3}}\left(\frac{1}{Q_{1}}+\frac{1}{Q_{2}}+\frac{1}{Q_{3}}\right), \\
& J_{R}^{\mathrm{BH}} \equiv J_{1}^{\mathrm{BH}}+J_{2}^{\mathrm{BH}}=\frac{2}{\sqrt{m}}\left(a_{1}-a_{2}\right) \sqrt{Q_{1} Q_{2} Q_{3}} .
\end{aligned}
$$

\subsection{Comparing the microstates and the black hole}

As we explained in the Introduction, extremal black holes have an infinite throat, and comparing the length of this throat to that of the fuzzballs is meaningless. Comparing the thicknesses of the throats on the other hand gives automatically the same result: the thickness is only controlled by the charges. For near-extremal black holes the thickness is also largely controlled by the charges, so it will automatically be the same for fuzzballs and black holes. On the other hand, non-extremal black holes have a finite throat, and hence comparing the lengths of the throats is now meaningful, and can indicate which fuzzballs are expected to be more typical than the others, and whether fuzzballs differ from the black hole away from the horizon microscopically or macroscopically.

We denote the difference in the length of the non-extremal black hole throat and that of its microstates by

$$
\Delta L \equiv L_{B H}-L_{M S}
$$

Although we have not backreacted the metastable bound state, we have argued above that a small probe supertube will not significantly change the geometry and hence $L_{M S}$ will be the length of the supersymmetric microstate throat given by (2.1). We can estimate the throat length by integrating along the $z$-axis, from the outermost center $z_{M S} \equiv z_{7}$ up to a suitable cutoff scale $z_{\text {neck }}$. The depth of the black hole throat is the metric distance from the horizon at $\rho=\rho_{+}$to the end of the throat at $\rho=\rho_{\text {neck }}$ with the metric (B.1). A suitable cutoff is $z_{\text {neck }}=\rho_{\text {neck }}=\left(Q_{1}^{B H} Q_{2}^{B H} Q_{3}^{B H}\right)^{1 / 6}$. The expression for $\Delta L$ is quite 
complicated, but as we explain in appendix $\mathrm{C}$ we can make a very insightful approximation through which we obtain

$$
\Delta L \approx \rho_{\text {neck }} \ln \left(2 \frac{\rho_{M S}}{\rho_{+}}\right),
$$

where we replaced the cutoff $z_{M S}$ by $\rho_{M S}$ in a spherically symmetric approximation of the microstate geometry.

Consider the following scaling of the supertube charges and of the coordinates of the GH centers:

$$
\begin{aligned}
\left(q_{1}, q_{2}, d_{3}\right) & \rightarrow e^{\lambda}\left(q_{1}, q_{2}, d_{3}\right), \\
\rho_{M S} & \rightarrow e^{\mu} \rho_{M S} .
\end{aligned}
$$

The approximated difference in depths then goes as

$$
\frac{\Delta L}{\rho_{\text {neck }}} \rightarrow \frac{\Delta L}{\rho_{\text {neck }}}-\frac{1}{4} \lambda+\frac{3}{4} \mu .
$$

This reveals that the black hole throat can be made deeper than that of the microstate ( $\Delta L$ positive) by taking either smaller tubes or deeper background microstates.

To confirm this approximation, we evaluate $\Delta L=L_{B H}-L_{M S}$ numerically. We do this for supertubes of charges

$$
\left(q_{1}, q_{2}, d_{3}\right)=e^{\lambda}(10,-50,1),
$$

with $\lambda=-10,-9, \ldots, 9,10$. The supertubes are placed in the nine background scaling geometries of different sizes of table 1 . The size of the black hole throat $L_{B H}$ is calculated from (C.3) for the rotating black hole geometry (B.1), and the parameters of the black hole are extracted from the metastable supertube minima as in section 4.2. The size of the microstate throat is obtained by integrating (C.1). We replace the background microstate geometry by that of the extremal black hole.

We plot our findings in figures $9 \mathrm{a}$ and $9 \mathrm{~b}$. In figure $9 \mathrm{a}$ we show the effect of scaling the tube charges. We plot $\Delta L$ for tubes of various sizes $(\lambda=-10,-9, \ldots, 9,10)$, in three scaling solutions of table 1 . We find all possibilities: microstates that are deeper, of the same depth, and shallower than the black hole. By making the tubes smaller, the black hole can always be made deeper than the microstate. It is also clear that $\Delta L$ has the scaling behavior anticipated in (4.15).

In figure $9 \mathrm{~b}$ we show the effect of putting the tube in backgrounds of different scaling size and depth. We plot $\Delta L$ for tubes in all nine scaling solutions (2,6 and 9 of table 1$)$, in terms of $\mu \equiv \log z_{7}$, where $z_{7}$ is the position of the outermost center of the scaling background. This reveals that the approximately linear scaling (4.15) still holds.

\subsection{The range of validity of our construction}

Having obtained non-extremal microstates by placing probe supertubes inside long supersymmetric fuzzballs, it is important to study the ranges of charges in which our construction is valid. Clearly, since we have not backreacted the supertube, and treated them as probes, 


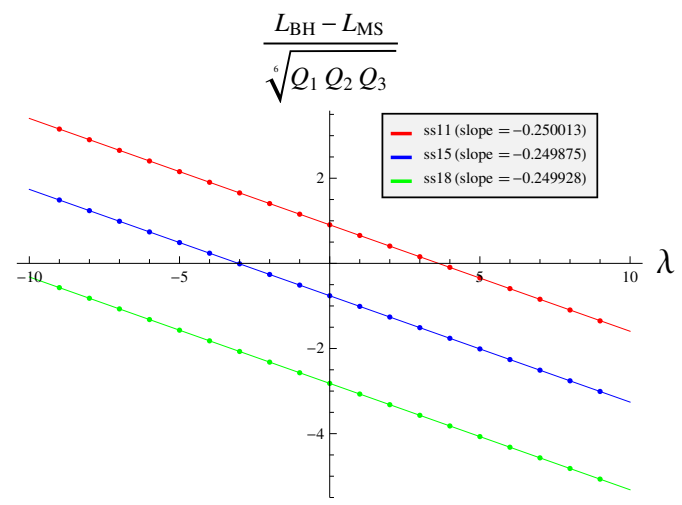

(a) The difference in depths $\frac{\Delta L}{\rho_{\text {neck }}}$ in terms of the tube charge scaling sizes $\left(q_{1}, q_{2}, d_{3}\right)=$ $e^{\lambda}(10,-50,1)$ for the backgrounds 2,6 and 9 of table 1 .

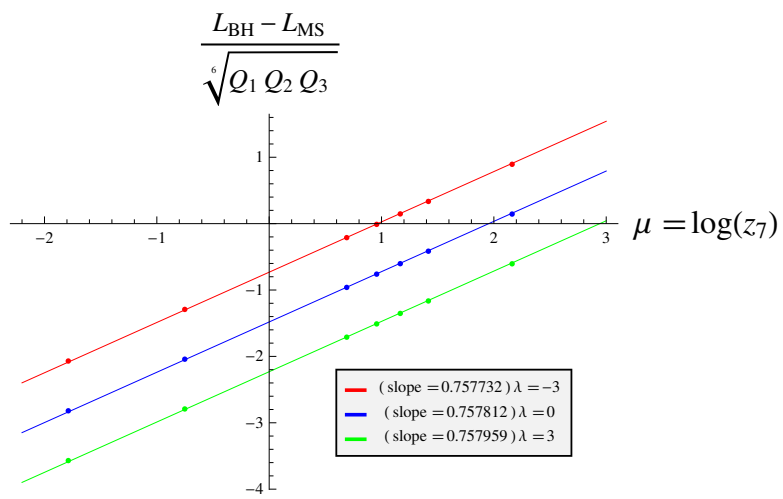

(b) The difference in depths $\frac{\Delta L}{\rho_{\text {neck }}}$ in terms of the scaling solution sizes measured by the logarithm of the position of the $7^{\text {th }}$ center $z_{7}$.

Figure 9. The difference in depths $\Delta L=L_{B H}-L_{M S}$ for tubes of size $\lambda=-10,-9, \ldots, 9,10$ in several scalings backgrounds.

we are automatically assuming that their charges and dipole charges are much smaller than those of the GH centers and we are only describing configurations whose mass above extremality is much smaller than the sum of the charges. These correspond to microstates of near-extremal black holes.

A possible mechanism for invalidating our construction is if the microstates we create will have closed timelike curves. In the absence of backreaction one cannot say precisely when or whether this will happen; however, one can estimate whether the angular momentum of a microstate is larger or smaller than the angular momentum that would cause closed timelike curves in a black hole of identical charges and length.

The non-extremal black hole geometry has closed timelike curves unless $m \geq\left(a_{1} \pm a_{2}\right)^{2}$. This gives two 'cosmic censorship bounds' on the black hole angular momenta. For a nearextremal black hole, the angular momenta (4.11) have to satisfy:

$$
\begin{aligned}
& J_{L}^{\mathrm{BH}} \leq \frac{m}{2} \sqrt{Q_{1} Q_{2} Q_{3}}\left(\frac{1}{Q_{1}}+\frac{1}{Q_{2}}+\frac{1}{Q_{3}}\right), \\
& J_{R}^{\mathrm{BH}} \leq 2 \sqrt{Q_{1} Q_{2} Q_{3}} .
\end{aligned}
$$

The second bound is automatically satisfied because we are starting with a BPS microstate of a black hole with a large horizon area, and adding a probe supertube only changes the charges and $J_{R}$ by very small amounts.

The first bound is more problematic. Since the BPS microstate has $J_{L}=0$, the resulting metastable microstate will get its left-moving angular momentum entirely from the $\vec{E} \times \vec{B}$ interactions between the supertube and the background. If we call this contribution $J_{L}^{\text {tube }}$ and express the parameter $m$ in terms of the energy of the metastable supertube 
$\Delta M$, this bound becomes

$$
J_{L}^{\text {tube }} \leq A(Q) \sqrt{\Delta M}, \quad A(Q)=\sqrt{2} \sqrt{Q_{1} Q_{2} Q_{3}} \sqrt{\frac{1}{Q_{1}}+\frac{1}{Q_{2}}+\frac{1}{Q_{3}}} .
$$

Since both the angular momentum $J_{L}^{\text {tube }}$ and $\Delta M$ scale linearly with the tube charges, we see that in a solution of fixed length this condition will be violated when the tube charges become very large.

Alternatively, one can consider a supertube with fixed charges in a solution whose length is dialed by hand by bringing the centers together on the GH base. The mass above extremality $\Delta M$ is linear in the inter-center separation, while we expect (from the known supersymmetric solutions) that the $\vec{E} \times \vec{B}$ interactions that give rise to $J_{L}^{\text {tube }}$ will remain constant. Hence, a solution with a single supertube that becomes too deep will start having charges and angular momenta outside of the cosmic censorship bound, and will most likely have closed timelike curves.

Of course, the way to avoid all these complications is to use the fact that the original solution is $\mathbb{Z}_{2}$-symmetric and place two identical supertubes in metastable minima symmetric around the origin, such that resulting configuration preserves this symmetry. In such a symmetric configuration the contribution to $J_{L}^{\text {tube }}$ from the interaction of the supertubes with the background vanishes, and the cosmic censorship conditions are always satisfied.

\subsection{The force on probe branes}

Another quantity that one can compute in both the near-extremal black hole geometry and in the non-extremal fuzzballs we construct is the force on a probe brane whose charge is carried by the black hole. Of course, this force is identically zero in the BPS black hole and in the BPS fuzzballs, but now we have more mass than charge, and we expect such a probe brane to start feeling a force.

The potential for an M2 brane wrapping the $I^{\text {th }}$ torus $T_{I}^{2}$, in the Cvetic-Youm black hole (appendix B) is easy to compute ${ }^{6}$

$$
V_{B H}^{(I)}=V_{D B I}+V_{W Z}=\frac{1}{H_{I}}\left(\sqrt{H_{m}}-\operatorname{coth} \delta_{I}\right) .
$$

As before, in the near-extremal limit with no rotation $Q_{I}^{B H} \approx Q_{I}$ and $m \ll Q_{1}$. The leading-order contribution to the potential of a probe M2 brane wrapping the torus $T_{I}^{2}$ is proportional to what we may define as the mass above extremality in the $I^{\text {th }}$ channel:

$$
V_{B H}^{(I)}=\frac{\Delta M_{I}}{\rho^{2}}, \quad \text { where } \quad \Delta M_{I} \equiv \frac{m^{2}}{8 Q_{I}} \quad \text { and } \quad \Delta M=\sum_{I=1}^{3} \Delta M_{I}+\mathcal{O}\left(m^{3}\right) .
$$

Note that probe branes wrapping different tori will correspond upon compactification to five dimensions to point particles with different types of $\mathrm{U}(1)$ charges, and feel different forces.

\footnotetext{
${ }^{6}$ The force is $\vec{F}_{B H}=q_{M 2} \frac{\partial V_{B H}}{\partial \vec{r}_{M 2}}$.
} 
To compute the force on a probe M2 brane in the non-extremal fuzzball one may think naively that one needs to construct the fully backreacted solution corresponding to metastable supertubes, but this is not so. Using the fact that in the absence of a metastable supertube one can add a BPS M2 brane at a large distance away from the throat without breaking supersymmetry, one can calculate the action of a metastable supertube in a microstate geometry both with and without the brane. The difference between the two actions gives then by Newton's third law the potential felt by far-away M2 brane as a function of its position, which can then be used to determine the force it feels. In the examples where a backreacted solution exists, this method, first introduced in KKLMMT [49], reproduces correctly the force computed from supergravity [55-58].

Adding an M2 brane with charge $q_{M 2}$ far away from the scaling centers introduces another term in the M2 harmonic function

$$
L_{I}=\sum_{i=1}^{7} \frac{\ell_{i}}{\left|\vec{r}-\vec{r}_{i}\right|}+\frac{q_{M 2}}{\left|\vec{r}-\vec{r}_{M 2}\right|},
$$

and for small charges this changes the energy of the metastable supertube by

$$
V_{M S}^{(I)}=\left.\frac{\partial \mathcal{H}}{\partial L_{I}}\right|_{\min } \frac{1}{\left|\vec{r}_{\min }-\vec{r}_{M 2}\right|},
$$

which by Newton's third law gives then the potential felt by the M2 brane in the nonextremal fuzzball.

Given that our non-extremal microstates have the same mass and charges as a nonextremal black hole, we would expect by Birkhoff's theorem that the leading-order term in the potential felt by an M2 brane far away from the region of the throat would be the same. However, the leading-order term in (4.22) is not of the same form as (4.20); in particular the microstate attractive potential (4.22) does not scale properly with the length of the microstate throat:

$$
\frac{V_{M S}^{(I)}}{V_{B H}^{(I)}} \sim \frac{1}{L_{I}}
$$

Since $1 / L_{I}$ is linear in the inter-center distances of the scaling background, the force with which the microstate attracts the M2 brane vanishes as one considers deeper and deeper microstates with the same mass. Furthermore, another surprise is in store. A microstate with a metastable supertube with electric charges $q_{1}$ and $q_{2}$ will attract M2 branes with charges $Q_{1}$ and $Q_{2}$, but will repel M2 branes with charge $Q_{3}$. This can be seen both by investigating (4.22), or by evaluating the potential numerically:

$$
\frac{V_{M S}^{(1)}}{V_{B H}^{(1)}} \approx 3.0 \times 10^{-5} z_{6}, \quad \frac{V_{M S}^{(2)}}{V_{B H}^{(2)}} \approx 2.9 \times 10^{-5} z_{6}, \quad \frac{V_{M S}^{(3)}}{V_{B H}^{(3)}} \approx-6.4 \times 10^{-5} z_{6} .
$$

The "wrong" sign of $V_{M S}^{(3)}$ and the linear dependence of these ratios on $z_{6}$ implies that one cannot hope to obtain the "correct" black-hole force on probe M2 branes from microstates constructed this way. One can also imagine constructing other types of black 
hole microstates, by placing single anti M2-branes or other more complicated objects inside bubbling geometries; however, the "force problem" persists, and it can be summarized as follows.

Given a background that does not attract M2 branes, the mass above extremality generated by adding a probe that breaks supersymmetry inside a long throat generically goes like the mass of that probe divided by the warp factor at the bottom of the throat. On the other hand, if one computes "à la KKLMMT" the force on a probe M2 brane, this force scales generically like the mass of the probe divided by the square of the warp factor, and hence like the ADM mass of the solution divided by the warp factor at the bottom. If one now makes the throat longer or shorter keeping the mass fixed, the force in a microstate changes, unlike in a black hole solution where this force is always proportional to the mass above extremality.

This force analysis indicates that the non-extremal microstates obtained by placing single metastable supertubes inside BPS microstates do not attract M2 branes in the way one may naively expect of a typical black hole microstate. The underlying reason for this is that the supertube couples not only to the warp factor and electric fields but also to extra scalars in five dimensions, which come from the volume moduli of the torus. This extra interaction, which is absent for M2 probes in the background of the black hole, leads to the different scaling behavior of the force on a probe M2 in the microstate background and to the repulsive force felt by an M2-brane along the third torus.

Even if the microstates we obtain by placing one metastable supertube do not attract M2 branes the way the black hole does, one can clearly bypass this problem and construct very large numbers of microstates that attract M2 branes typically by placing several species of metastable supertubes, and by fixing the supertube charges such that the length of the microstate is exactly that of the black hole (4.15) and furthermore such that at this length the forces are exactly those of the black hole (4.22). However, this is more a matter of engineering, and can obscure an important piece of physics that the force computation reveals: the fact that the force on a probe M2 branes varies wildly from microstate to microstate, and can be even negative, implies that M2 branes will feel the thermal fluctuations between various fuzzballs as thermal fluctuations in the force even if they are quite far away from the black hole. Thus, these M2 branes will become aware of the existence of fuzzballs and of the breakdown of classical physics further away from the horizon than other probes.

\section{Fuzzballs of fire or fuzzballs of fuzz (in lieu of conclusions)}

In this paper we have used probe supertubes to construct microstate geometries, or fuzzballs, that have the same mass and charges as three-charge non-extremal black holes. We computed the length of the throats of these solutions, and found that one can easily build microstates whose throats are longer, shorter or have the same length as the throat of the black hole. Since in our construction there is no dynamical mechanism that sets the microstate length to be the same as that of the black hole throat, this indicates that this mechanism may be entropic: there will be many more microstates of black hole throat 
length than shorter or longer ones. Of course, to produce such an entropic argument one needs first to make sure that our method for constructing non-extremal microstates can produce at least a subset of the typical ones, and them count these microstates.

However, the absence of a dynamical mechanism for fixing the microstate length indicates that fuzzballs will differ from the black hole at macroscopic distances from the horizon, and not just in its vicinity (as recently mentioned as a possibility in [30]). Of course, this intuition applies to near-extremal fuzzballs, and the extrapolation to more generic black holes may break down. Nevertheless, one can use near-extremal black holes as a testing ground for all the ideas proposed in relation to infalling observer physics, firewalls, black hole complementarity and spacelike singularity resolution and this is the purpose of this section.

\subsection{What does an in-falling observer see?}

The first question one can try to address is the scale at which an infalling observer stops experiencing spacetime. In the most straightforward interpretation of the fuzzball proposal, which one may call a "fuzzball of fire" interpretation, the classical geometry breaks down at the horizon, and is replaced by an ensemble of fuzzballs [2]. Hence, the horizon is the scale where the "thermodynamic" description of physics (in terms of a classical spacetime) breaks down, and the "statistical" description (in terms of fuzzballs) takes over. In a naive analogy with an ideal gas, the scale of the horizon is like that of the mean free path, and hence we might expect the incoming observers to experience large statistical fluctuations in the same way in which a particle of smaller and smaller size in a gas experiences larger and larger fluctuations. Below a certain size of the particle the Brownian motion deviations overtake the classical trajectory, and the notion of "particle moving in a continuous fluid" breaks down. In the same way, a particle far away from a black hole experiences a classical spacetime, but as the particle approaches the horizon the statistical fluctuations become stronger and stronger, and at the horizon the notion of "particle moving in a classical spacetime" breaks down.

From the point of view of the incoming particle, the increasing fuzzball fluctuations it feels at the scale of the horizon are not a very pleasant experience, which agrees with the recent proposal of [30] that an incoming particle must see a firewall at the horizon scale in order for the information paradox to be solved. In [36] one of the authors and Chowdhury have argued that the pleasantness an in-falling particle experiences depends on its energy; particles of the order of the Hawking radiation should thermalize in the bath of out-going radiation which thus constitutes a firewall for these in-falling particles, while particles much heavier than Hawking radiation should pass the bath nearly unaltered.

Recently Mathur has argued for a new approximate complementary for observers heavier than Hawking radiation falling into a fuzzball. According to this "fuzzball complementarity" paradigm [35, 59, 60], which draws from recent ideas of [61, 62], the scale of fuzzball thermalization/loss of spacetime experience does not depend only on the location of the infalling observer, but also on its energy. Heavier observers will continue experiencing a spacetime even after they have passed the horizon scale and have entered the fuzzball region, and for these observers spacetime will emerge from the quantum superposition of the 
fuzzballs. On the other hand lighter particles, of order the Hawking radiation energy, stop experiencing a classical spacetime at the horizon - this is necessary in order for the Hawking radiation to be able to carry off the black hole information to infinity and solve the information paradox. According to the "fuzzball complementarity" paradigm, the analogy with the ideal gas in the "fuzzball of fire" argument above is not so straightforward, essentially because in the ideal gas there is only one scale, while when describing an observer falling into a black hole one has two scales: the observer mass and its location.

It is hard to tell directly whether our non-extremal fuzzballs will be felt by an incoming observer as fuzzballs of fire or as fuzzballs of fuzz (in the sense of fuzzball complementarity). To do this one would have to construct first more generic non-extremal fuzzballs, and then to scatter various particles off them. Such a research programme is feasible; one can in particular use the quiver quantum mechanics that describes these fuzzballs in the regime of parameters where gravity is turned off [45], and analyze the scattering of various charged centers, as one does in supergoop studies [63]. One can analyze for example the collision of a multicenter near-extremal fuzzball goop with a center whose charges are much bigger than those of the centers that compose the fuzzball, and see whether such a center gets absorbed by the fuzzball goop as soon as it reaches it or traverses it with impunity, and if so how does the trajectory of this center differ from the trajectory in a single-center black hole geometry.

However, even before such a calculation is done, there are two features of our construction that are relevant in the fuzzballs of fire/fuzz discussion. First, the fact that nothing dramatic happens as the throat of microstate geometries becomes longer or shorter than that of a black hole implies that the difference between fuzzballs and the classical black hole is not strongly suppressed immediately above the horizon scale. Hence, if an observer heavier than Hawking particles continues to experience a spacetime below the horizon scale then, by extension, an observer lighter than the Hawking particles should stop experiencing a spacetime above the horizon scale. The physics of this possibility can get quite unpalatable: a spaceship orbiting at say five Schwarzschild radii above the horizon cannot send to another nearby spaceship any photons that have an energy lower than the Hawking radiation energy divided by five to some power; such photons are thermalized by the ensemble of fuzzballs already at that scale and hence cannot propagate.

Since we do not expect observers, be they very small, to start experiencing large statistical fluctuations of spacetime and dissolve in the fuzz far away from the horizon, this Gedanken experiment seems to tilt the balance against "fuzzball complementarity", and towards the "fuzzball of fire" interpretation. On the other hand, another piece of fuzzball physics in our construction seems to incline the balance backwards:

We have computed the force on probe M2 branes (that experience no force when the fuzzball is supersymmetric), and we have found that this force varies wildly, and can even change sign when one goes from one metastable fuzzball to another. It may be that this wild variations in the force are just a feature of the very specific type of fuzzballs we have succeeded to construct, and some yet-to-be-constructed more typical microstates will not attract M2 branes in such erratic ways. However, it is also possible that we have uncovered a fundamental feature of fuzzballs of near-extremal black holes: they may attract very erratically the components of the extremal black hole with the same charges. 
Now, if these very special probes see the thermal noise from the fuzzball and experience statistical fluctuation already at a very large distance, way above the horizon scale, it does not seem so far-fetched that other observers, in particular those with energy below that of Hawking radiation, could also see this thermal noise far away from the horizon, while other (more massive) observers continue experiencing a spacetime well into the fuzzball, as the "fuzzball complementarity" paradigm indicates.

\subsection{Is Hawking radiation coming from brane-flux annihilation?}

Besides being the first examples of non-extremal fuzzballs and maybe a way to realize firewalls in string theory, one can also use our configurations to explore other pieces of black hole physics. The first is Hawking radiation. The metastable supertubes we use to construct the fuzzballs decay into supersymmetric vacua via brane-flux annihilation [42, 43] and this decay corresponds to the near-extremal black hole emitting its last Hawking radiation quantum and becoming an extremal black hole. This process is quite difficult to study from the black hole side, essentially because thermodynamics breaks down [64, 65]. A comparison of the fuzzball decay rates (which one can compute rather straightforwardly) to the near-extremal black hole emission rate may shed light on how thermodynamics breaks down, and also on which fuzzballs are more typical than others: the decay rates depend on how big the bubbles of a fuzzball are, and can be used to determine the typical bubble size.

The other important question is what is the backreacted solution corresponding to our metastable supertubes. The JMart solution [37], which is one of the two known fullybackreacted non-extremal fuzzballs, is known to have ergoregions, and its instability [66] has been argued [67] to correspond to Hawking radiation. The other fully-backreacted non-extremal fuzzball, the running-Bolt solution [40], is also unstable, but its instability does not come from ergoregions. Our configurations on the other hand are metastable, at least in the probe approximation. It might be possible that the energy of some metastable supertubes will decrease by taking one of the GH centers off the symmetry axis, and if this happens the fully backreacted solution will probably be unstable as well. However, it is also very likely that one will be able to construct metastable supertubes that remain metastable when fully backreacted, and thus will have very different physics from the JMaRT and running-Bolt solutions.

The other issue with the metastable supertube backreaction is that most of the work analyzing the backreaction of antibranes in backgrounds with charge dissolved in fluxes indicates that such backreacted solutions have unphysically-looking singularities, both at first order [56, 57, 68-71] and when looking at the fully-backreacted solutions [72, 73]. If one would naively extend this result to our work, one might expect that anti-M2 branes in long BPS throats will also give rise to unphysically-looking singularities. However, in our construction we are not using bare antibranes, but supertubes that carry two kinds of antibrane charges, and also have a dipole charge and a nonzero angular momentum. The advantage of supertubes is that if they are solutions of the DBI Hamiltonian they backreact into geometries that are smooth in the D1-D5-P duality frame [46]. Hence we expect the backreaction of our metastable supertubes to give rise to regular solutions; it 
would be very interesting to confirm this by constructing directly this challenging nonsupersymmetric cohomogeneity-two solution.

\subsection{Are spacelike singularities resolved backwards in time?}

The existence of microstate solutions that have the same mass, charges and throat length as non-extremal black holes indicates that the singularity of these black holes will most likely not only be resolved to the inner horizon (as one may expect by extrapolating the extremal black hole result) but all the way to the outer one, which is backwards in time from where the singularity is, as the Penrose diagrams in figure 1 show. One can now try to see what this intuition may tell us about singularity resolution.

Indeed, since the Penrose diagram of the near-extremal black holes is the same as that of all Reissner-Nordström black holes, one can extrapolate our result and assume that the singularity of all Reissner-Nordström black holes is resolved all the way to the outer horizon. One can then take the small charge limit (in which the inner horizon and the timelike singularity merge to form a spacelike singularity) and infer that the spacelike singularity of the zero-charge Schwarzschild black hole is also resolved backwards in time, all the way to the horizon.

If this is indeed the correct pattern of the resolution of spacelike singularities in string theory, one can ask two questions:

1. What is the mechanism by which this happens and the corresponding scale?

2. What does this imply for the physics of other spacelike singularities, like the cosmological ones?

Both questions have several possible answers, and we leave it to the reader who is unhappy with them to find more compelling ones. To answer Question 1, one can always argue that singularities in string theory have low-mass degrees of freedom, that destroy the spacetime on macroscopic distances. One can also refine the answer, and argue [74] that an incoming shell that will form such a singularity in the future will enter in a region where there are a very large number of fuzzball-like states, and even if the probability of tunneling into any of them is tiny, since there are so many of them, the incoming shell will tunnel in the fuzz with probability one. The size of the region where the singularity is resolved depends on the mass of the singularity, and on the density of fuzzballs.

The answer to the second question depends largely of the mechanism for the backwards in time resolution. If this mechanism involves tunneling into fuzzball-like configurations that live near say a Big Crunch singularity, then the scale for the resolution will probably be given by the typical size of these configurations (which is quite hard to estimate at this point, in the absence of any explicit Big-Crunch-resolving fuzzballs). Furthermore, one can argue that such configurations were also present in the early universe, where they can again be thought of as giving a "forward in time" resolution of the Big Bang singularity, and their physics might have cosmological implications $[75,76]$. We leave the fascinating exploration of these possibilities to future work. 


\section{Acknowledgments}

We would like to thank Borun D. Chowdhury, Samir Mathur, Thomas Van Riet and Nick Warner for useful discussions. This work was supported in part by the ANR grant 08-JCJC0001-0 and by the ERC Starting Independent Researcher Grant 240210 - String-QCD-BH. BV would like to thank Evelien Dejonghe for her support.

\section{A Smooth scaling backgrounds}

We focus on three-charge backgrounds that are microstate geometries of black holes and black rings. Microstate geometries are everywhere smooth and free of horizons, such that each individual geometry carries no entropy. They have the same mass, charges and angular momenta as their black hole or black ring counterpart. Deep microstate geometries have a scaling behavior: the centers can be put arbitrarily close such that the geometry develops a very long throat, while the curvature is small everywhere.

\section{A.1 Smoothness and regularity}

The first physical requirement on the background is the absence of closed timelike curves (CTC's) in the geometry, giving the necessary conditions: ${ }^{7}$

$$
Z_{1} Z_{2} Z_{3} V-\mu^{2} V^{2} \geq 0 \quad \text { and } \quad V Z_{I} \geq 0 .
$$

Note that this ensures that the potential (2.7) is well-defined (radicand under square root is positive). To have a smooth geometry, the warp factors and the function $\mu$ appearing in the angular momentum one-form $k$ must be regular at the sources of the harmonic functions. This yields relations between the charges and the constants in the harmonic functions. The constants are further constrained by demanding asymptotic flatness. We will choose the harmonic functions $V$ (Taub-NUT charges) and $K^{I}$ (dipole charges) to be fixed as:

$$
V=\sum_{j=1}^{N} \frac{v_{j}}{r_{j}}, \quad K^{I}=\sum_{j=1}^{N} \frac{k_{j}^{I}}{r_{j}}
$$

Regularity requires these harmonic functions to be sourced at the same points and one must take $v_{j} \in \mathbb{Z}$. For the base metric to be asymptotically $\mathbb{R}^{4}$ one must impose

$$
\sum_{j=1}^{N} v_{j}=1
$$

Then smoothness determines $L_{I}$ (M2 charges) and $M$ (momentum along $\psi$ ) to be

$$
L_{I}=1-\frac{1}{2} C_{I J K} \sum_{j=1}^{N} \frac{k_{j}^{J} k_{j}^{K}}{v_{j}} \frac{1}{r_{j}}, \quad M=m_{0}+\frac{1}{12} C_{I J K} \sum_{j=1}^{N} \frac{k_{j}^{I} k_{j}^{J} k_{j}^{K}}{v_{j}^{2}} \frac{1}{r_{j}},
$$

\footnotetext{
${ }^{7}$ The sufficient no-CTC condition, which insures the existence of a time function is $Z_{1} Z_{2} Z_{3} V-\mu^{2} V^{2} \geq$ $\omega^{2}[8]$.
} 


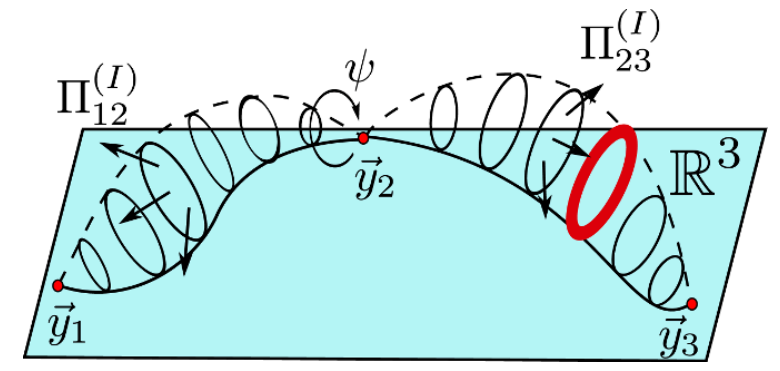

Figure 10. Smooth three-charge bubbling geometry with a supertube (red) placed on one of the cycles along $\psi$.

with

$$
m_{0}=-\frac{1}{2} \frac{\sum_{j=1}^{N} \sum_{I=1}^{3} k_{j}^{I}}{\sum_{i=1}^{N} v_{i}}=-\frac{1}{2} \sum_{j=1}^{N} \sum_{I=1}^{3} k_{j}^{I} .
$$

After imposing regularity and smoothness as well as asymptotic flatness there is a residual freedom in choosing $N-1$ Taub-NUT charges and $N$ dipole charges.

The microstates are 'bubbled' geometries. For $N$ centers, there are $N-1$ non-trivial two-cycles, or bubbles, on the GH base. The cycles are supported by $N-1$ non-trivial fluxes $\Pi_{i j}^{(I)}$ :

$$
\left.\Pi_{i j}^{(I)} \equiv \frac{K^{I}}{V}\right|_{r_{j}}-\left.\frac{K^{I}}{V}\right|_{r_{i}}=\left(\frac{k_{j}^{I}}{v_{j}}-\frac{k_{i}^{I}}{v_{i}}\right) .
$$

We depict such a geometry in figure 10.

The smoothness condition together with the first condition of (A.1) leads to a further requirement that ensures the absence of CTC's: $\mu$ has to vanish at each center, since for $r_{i} \rightarrow 0$ the $Z_{I}$ 's tend to finite values while $V^{-1}$ goes to zero. This gives $N-1$ bubble equations $[2,7,8,77]$. By writing the charges and constants in the harmonic functions as vectors $\Gamma_{i}=\left(v_{i}, k_{i}^{I}, \ell_{I, i}, m_{i}\right)$ and $h=\left(V_{\infty}, K_{\infty}^{I}, L_{I, \infty}, M_{\infty}\right)$ these are: ${ }^{8}$

$$
\forall i: \quad \sum_{\substack{j=1 \\ j \neq i}}^{N} \frac{\left\langle\Gamma_{i}, \Gamma_{j}\right\rangle}{r_{i j}}+\left\langle\Gamma_{i}, h\right\rangle=0
$$

where $\left\langle\Gamma_{i}, \Gamma_{j}\right\rangle=v_{i} m_{j}-m_{i} v_{j}+\frac{1}{2}\left(k_{i}^{I} \ell_{I, j}-\ell_{I, i} k_{j}^{I}\right)$ is the symplectic product and $r_{i j}=\left|\vec{r}_{j}-\vec{r}_{i}\right|$ are the inter-center distances. For smooth solutions, the bubble equations can be written in terms of the magnetic two-form fluxes $\Pi_{i j}^{(I)}$ through the bubbles as:

$$
\frac{1}{6} C_{I J K} \sum_{\substack{j=1 \\ j \neq i}}^{N} \Pi_{i j}^{(I)} \Pi_{i j}^{(J)} \Pi_{i j}^{(K)} \frac{v_{i} v_{j}}{r_{i j}}=-2\left(m_{0} v_{i}+\frac{1}{2} \sum_{I=1}^{3} k_{i}^{I}\right) .
$$

The bubble equations relate the magnetic flux through each bubble to the physical size of each bubble.

\footnotetext{
${ }^{8}$ In more general solutions $[44,45,78]$ these equations come from imposing that $\omega$ should have no Dirac-Misner strings at the centers, but in smooth backgrounds this is equivalent to (A.7).
} 


\section{A.2 Asymptotic charges and angular momenta}

We give the charges and angular momenta of the five-dimensional solutions. Since the solution is invariant under the gauge transformation $K^{I} \rightarrow K^{I}+c^{I} V$, or $k_{j}^{I}+c^{I} v_{j}$, for any constant $c^{I}$, we define asymptotic quantities in terms of the gauge invariant flux parameters:

$$
\tilde{k}_{j}^{I} \equiv k_{j}^{I}-v_{j}\left(\sum_{j=1}^{N} k_{j}^{I}\right) .
$$

The electric charges of the solution as measured at infinity are extracted from the $\rho^{-2}$ term, with $r=\frac{1}{4} \rho^{2}$ in the expansion of the warp factors $Z_{I}:^{9}$

$$
Q_{I}=-2 C_{I J K} \sum_{j=1}^{N} \frac{\tilde{k}_{j}^{J} \tilde{k}_{j}^{K}}{v_{j}} .
$$

In five dimensions there are two angular momenta, which are read off from the asymptotic behavior of $k$ in (2.5) from the terms that have a $\rho^{-2}$ fall-off:

$$
k \sim \frac{1}{4 \rho^{2}}\left(\left(J_{1}+J_{2}\right)+\left(J_{1}-J_{2}\right) \cos \theta\right) d \psi+\ldots
$$

where $\theta$ is the angle between $\vec{r}$ and the dipoles $\vec{D} \equiv \sum_{j=1}^{N} \sum_{I=1}^{3} \tilde{k}_{j}^{I} \vec{r}_{j}$. The two angular momenta are then given by

$$
J_{R} \equiv J_{1}+J_{2}=\frac{4}{3} C_{I J K} \sum_{j=1}^{N} \frac{\tilde{k}_{j}^{I} \tilde{k}_{j}^{J} \tilde{k}_{j}^{K}}{v_{j}^{2}} \quad \text { and } \quad J_{L} \equiv J_{1}-J_{2}=8|\vec{D}| .
$$

Using the bubble equations, one can associate an angular momentum flux vector with the $i j^{\text {th }}$ bubble:

$$
\vec{J}_{L}=\sum_{i>j} \vec{J}_{L, i j}, \quad \vec{J}_{L, i j} \equiv-\frac{4}{3} v_{i} v_{j} C_{I J K} \Pi_{i j}^{(I)} \Pi_{i j}^{(J)} \Pi_{i j}^{(K)} \frac{\left(\vec{r}_{i}-\vec{r}_{j}\right)}{\left|\vec{r}_{i}-\vec{r}_{j}\right|}
$$

The flux on the left-hand side of the bubble equation (A.8) yields the contribution of the bubble to $J_{L}$.

\section{B Non-extremal black hole geometry}

The non-extremal rotating black hole solution sourced by three M2's on $T^{6}$ is the CveticYoum black hole. We give it in the notation of [79]. The solution depends on six parameters: a mass parameter $m$, three 'boosts' $\delta_{I}$ related to the charges and angular momentum parameters $a_{1}, a_{2}$. The metric is

$$
d s_{11}^{2}=-\left(H_{1} H_{2} H_{3}\right)^{-2 / 3} H_{m}(d t+k)^{2}+\left(H_{1} H_{2} H_{3}\right)^{1 / 3} d s_{4}^{2}+\sum_{I=1}^{3} \frac{\left(H_{1} H_{2} H_{3}\right)^{1 / 3}}{H_{I}} d s_{I}^{2} .
$$

\footnotetext{
${ }^{9}$ To isolate the charges of the solution one needs to take (2.3) to a standard polar form for $\mathbb{R}^{4}$ via $r=\frac{1}{4} \rho^{2}$.
} 
with

$$
k=\frac{m}{f}\left[-\frac{c_{1} c_{2} c_{3}}{H_{m}}\left(a_{1} \cos ^{2} \theta d \psi+a_{2} \sin ^{2} \theta d \phi\right)+s_{1} s_{2} s_{3}\left(a_{2} \cos ^{2} \theta d \psi+a_{1} \sin ^{2} \theta d \phi\right)\right]
$$

with $I, J, K$ all different and we write

$$
c_{I} \equiv \cosh \delta_{I}, \quad s_{I} \equiv \sinh \delta_{I} .
$$

The solution is built from the functions

$$
H_{I}=1+\frac{m s_{I}^{2}}{f}, \quad H_{m}=1-\frac{m}{f}, \quad f=\rho^{2}+a_{1}^{2} \sin ^{2} \theta+a_{2}^{2} \cos ^{2} \theta .
$$

The four-dimensional metric is

$$
\begin{aligned}
d s_{4}^{2}= & \frac{f \rho^{2}}{g} d \rho^{2}+f\left(d \theta^{2}+\sin ^{2} \theta d \phi^{2}+\cos ^{2} \theta d \psi^{2}\right) \\
& +H_{m}^{-1}\left(a_{1} \cos ^{2} \theta d \psi+a_{2} \sin ^{2} \theta d \phi\right)^{2}-\left(a_{2} \cos ^{2} \theta d \psi+a_{1} \sin ^{2} \theta d \phi\right)^{2}, \\
g= & \left(\rho^{2}+a_{1}^{2}\right)\left(\rho^{2}+a_{2}^{2}\right)-m \rho^{2} \equiv\left(\rho^{2}-\rho_{+}^{2}\right)\left(\rho^{2}-\rho_{-}^{2}\right) .
\end{aligned}
$$

The inner and outer horizon are given by the roots of $g(\rho)$ :

$$
\left(\rho_{ \pm}\right)^{2}=\frac{1}{2}\left(m-a_{1}^{2}-a_{2}^{2} \pm \sqrt{\left(m-a_{1}^{2}-a_{2}^{2}\right)^{2}-4 a_{1}^{2} a_{2}^{2}}\right) .
$$

The ADM mass, electric charges and angular momenta of the black hole are

$$
\begin{aligned}
M_{A D M} & =\frac{m}{2} \sum_{I} \cosh 2 \delta_{I}, & J_{1} & =m\left(a_{1} c_{1} c_{2} c_{3}-a_{2} s_{1} s_{2} s_{3}\right), \\
Q_{I} & =\frac{m}{2} \sinh 2 \delta_{I}, & J_{2} & =-m\left(a_{2} c_{1} c_{2} c_{3}-a_{1} s_{1} s_{2} s_{3}\right),
\end{aligned}
$$

where we have set $G_{5}=\frac{\pi}{4}$ as discussed in appendix A of [79].

\section{Approximation for throat depth}

We can give a good measure of the throat depth by integrating along the $z$-axis, from the outermost center $z_{M S} \equiv z_{7}$ up to a suitable cutoff scale $z_{\text {neck: }}$ :

$$
L_{M S} \equiv \int_{z_{M S}}^{z_{\text {neck }}} V^{1 / 2}\left(Z_{1} Z_{2} Z_{3}\right)^{1 / 6} d z
$$

The depth of the black hole throat is the metric distance from the horizon at $\rho=\rho_{+}$to the end of the throat at $\rho=\rho_{\text {neck }}$ which can be approximated by

$$
\rho_{\text {neck }}=\left(Q_{1}^{B H} Q_{2}^{B H} Q_{3}^{B H}\right)^{1 / 6} .
$$

The depth of the throat is then given by integrating $\sqrt{g_{\rho \rho}}$ in the metric (B.1):

$$
L_{B H} \equiv \int_{\rho_{+}}^{\rho_{\text {neck }}} \sqrt{g_{\rho \rho}} d \rho=\int_{\rho_{+}}^{\rho_{\text {neck }}} \frac{\rho \sqrt{f}}{\sqrt{g}}\left(H_{1} H_{2} H_{3}\right)^{1 / 6} d \rho .
$$


To get a feeling for $\Delta L$, we make some approximations. First, we approximate the geometry of the non-extremal black hole by a non-rotating one, so $a_{1}=a_{2}=0$. We get:

$$
L_{B H}=\int_{r_{+}}^{r_{\text {neck }}} \frac{\left(H_{1} H_{2} H_{3}\right)^{1 / 6}}{\sqrt{1-\frac{m}{\rho^{2}}}} d \rho
$$

with $H_{I}=1+Q_{I}^{B H} / \rho^{2}$. For near-extremal black holes, this is a good approximation. We also replace the microstate geometry by the (spherically symmetric) geometry of the extremal black hole metric:

$$
L_{M S}=\int_{r_{M S}}^{r_{\text {neck }}}\left(Z_{1} Z_{2} Z_{3}\right)^{1 / 6} \frac{d \rho}{\sqrt{r}}=\int_{\rho_{M S}}^{\rho_{\text {neck }}}\left(Z_{1} Z_{2} Z_{3}\right)^{1 / 6} d \rho,
$$

where we performed the change of variables $r=\frac{1}{4} \rho^{2}$ and we have $Z_{I}=1+Q_{I} / \rho^{2}$. This is a valid approximation, since the extremal black hole geometry only differs significantly from the microstate very deep down the throat.

Second, we approximate the black hole integral by splitting it into a part where $\rho_{\text {neck }}>$ $\rho \gg \rho_{+}$and a part where $\rho_{\text {neck }} \gg \rho>\rho_{+}$. We choose some intermediate radius $\rho_{\text {int }} \approx$ $\sqrt{\rho_{+} \rho_{\text {neck }}}$, but its exact value is of no importance. ${ }^{10}$ With this approximation C.4 becomes:

$$
L_{B H}=\rho_{\text {neck }} \int_{\rho_{+}}^{\rho_{\text {int }}} \frac{d \rho}{\sqrt{\rho^{2}-\rho_{+}^{2}}}+\int_{\rho_{\text {int }}}^{\rho_{\text {neck }}}\left(H_{1} H_{2} H_{3}\right)^{1 / 6} d \rho
$$

where we used that for the non-rotating non-extremal black hole the non-extremality parameter $m$ is just the square of the horizon radius $\rho_{+}$. In the same way we approximate C.5:

$$
L_{M S}=\rho_{\text {neck }} \int_{\rho_{M S}}^{\rho_{\text {int }}} \frac{d \rho}{\rho}+\int_{\rho_{\text {int }}}^{\rho_{\text {neck }}}\left(Z_{1} Z_{2} Z_{3}\right)^{1 / 6} d \rho
$$

Third, we know that for the extremal and non-extremal black hole the charges are almost equal because we are working with supertube probes, and hence also the $Z_{I}=H_{I}$ are equal. Then the difference in depth is

$$
\begin{aligned}
L_{B H}-L_{M S} & =\rho_{\text {neck }}\left(\int_{\rho_{+}}^{\rho_{\text {int }}} \frac{d \rho}{\sqrt{\rho^{2}-\rho_{+}^{2}}}-\int_{\rho_{+}}^{\rho_{\text {int }}} \frac{d \rho}{r}\right) \\
& =\rho_{\text {neck }}\left[\ln \left(\frac{\rho_{\text {int }}+\sqrt{\rho_{\text {int }}^{2}-\rho_{+}^{2}}}{\rho_{+}}\right)-\ln \frac{\rho_{\text {int }}}{\rho_{M S}}\right]
\end{aligned}
$$

Since we chose $\rho_{\text {int }} \gg \rho_{+}$, we can approximate this very well by

$$
\Delta L=L_{B H}-L_{M S}=\rho_{\text {neck }} \ln \left(2 \frac{\rho_{M S}}{\rho_{+}}\right) .
$$

\footnotetext{
${ }^{10}$ In our example, we have $\rho_{\text {neck }}^{2} \approx 10^{6}, \rho_{+}^{2} \lesssim 10^{2}$ and we can choose $\rho_{\text {int }}^{2} \sim 10^{4}$.
} 
We can use this result to get some idea on how the size of the supertubes and the depth of the microstates affect $\Delta L$. Consider the scaling of the supertube charges and the coordinates of the microstate centers as

$$
\begin{aligned}
\left(q_{1}, q_{2}, d_{3}\right) & \rightarrow e^{\lambda}\left(q_{1}, q_{2}, d_{3}\right), \\
\rho_{M S} & \rightarrow e^{\mu} \rho_{M S},
\end{aligned}
$$

Both scalings have a non-trivial effect on the size of the horizon radius of the would-be non-extremal black hole, which (neglecting rotation) is given by

$$
\rho_{+}^{2}=m=\sqrt{\frac{8 \Delta M}{\frac{1}{Q_{1}}+\frac{1}{Q_{2}}+\frac{1}{Q_{2}}}} .
$$

The potential $\mathcal{H}$ scales linearly with the tube charges, and in the scaling regime it also scales linearly with the coordinate size of the centers, see eq. (2.11). The same applies, of course, to the value $\Delta M$ of its metastable minimum. Hence the horizon radius, as a function of the tube charges $q^{\text {tube }} \equiv\left(q_{1}, q_{2}, d_{3}\right)$ and the size of the microstate background $\rho_{M S}$, has the following scaling behavior:

$$
\rho_{+}\left(q^{\text {tube }} ; \rho_{M S}\right)=e^{-(\lambda+\mu) / 4} \rho_{+}\left(e^{\lambda} q^{\text {tube }} ; e^{\mu} \rho_{M S}\right) .
$$

Therefore under the scalings (C.10), the difference in depths $\Delta L \equiv L_{B H}-L_{M S}$ goes as

$$
\frac{\Delta L}{\rho_{\text {neck }}} \rightarrow \frac{\Delta L}{\rho_{\text {neck }}}-\frac{1}{4} \lambda+\frac{3}{4} \mu \text {. }
$$

Open Access. This article is distributed under the terms of the Creative Commons Attribution License which permits any use, distribution and reproduction in any medium, provided the original author(s) and source are credited.

\section{References}

[1] S.D. Mathur, The Fuzzball proposal for black holes: An Elementary review, Fortsch. Phys. 53 (2005) 793 [hep-th/0502050] [INSPIRE].

[2] I. Bena and N.P. Warner, Black holes, black rings and their microstates, Lect. Notes Phys. 755 (2008) 1 [hep-th/0701216] [INSPIRE].

[3] S.D. Mathur, Fuzzballs and the information paradox: A Summary and conjectures, arXiv: 0810.4525 [INSPIRE].

[4] V. Balasubramanian, J. de Boer, S. El-Showk and I. Messamah, Black Holes as Effective Geometries, Class. Quant. Grav. 25 (2008) 214004 [arXiv:0811.0263] [INSPIRE].

[5] K. Skenderis and M. Taylor, The fuzzball proposal for black holes, Phys. Rept. 467 (2008) 117 [arXiv: 0804.0552] [INSPIRE].

[6] B.D. Chowdhury and A. Virmani, Modave Lectures on Fuzzballs and Emission from the D1 - D5 System, arXiv:1001.1444 [InSPIRE].

[7] I. Bena and N.P. Warner, Bubbling supertubes and foaming black holes, Phys. Rev. D 74 (2006) 066001 [hep-th/0505166] [INSPIRE]. 
[8] P. Berglund, E.G. Gimon and T.S. Levi, Supergravity microstates for BPS black holes and black rings, JHEP 06 (2006) 007 [hep-th/0505167] [INSPIRE].

[9] I. Bena, C.-W. Wang and N.P. Warner, Mergers and typical black hole microstates, JHEP 11 (2006) 042 [hep-th/0608217] [INSPIRE].

[10] V. Balasubramanian, E.G. Gimon and T.S. Levi, Four Dimensional Black Hole Microstates: From D-branes to Spacetime Foam, JHEP 01 (2008) 056 [hep-th/0606118] [INSPIRE].

[11] I. Bena, C.-W. Wang and N.P. Warner, Plumbing the Abyss: Black ring microstates, JHEP 07 (2008) 019 [arXiv: 0706.3786] [InSPIRE].

[12] I. Bena, N. Bobev, S. Giusto, C. Ruef and N.P. Warner, An Infinite-Dimensional Family of Black-Hole Microstate Geometries, JHEP 03 (2011) 022 [Erratum ibid. 1104 (2011) 059] [arXiv: 1006.3497] [INSPIRE].

[13] B.E. Niehoff, O. Vasilakis and N.P. Warner, Multi-Superthreads and Supersheets, arXiv:1203.1348 [INSPIRE].

[14] K. Goldstein and S. Katmadas, Almost BPS black holes, JHEP 05 (2009) 058 [arXiv: 0812.4183] [INSPIRE].

[15] I. Bena, S. Giusto, C. Ruef and N.P. Warner, Multi-Center non-BPS Black Holes: the Solution, JHEP 11 (2009) 032 [arXiv:0908.2121] [INSPIRE].

[16] I. Bena, S. Giusto, C. Ruef and N.P. Warner, Supergravity Solutions from Floating Branes, JHEP 03 (2010) 047 [arXiv:0910.1860] [INSPIRE].

[17] G. Dall'Agata, S. Giusto and C. Ruef, U-duality and non-BPS solutions, JHEP 02 (2011) 074 [arXiv: 1012.4803] [INSPIRE].

[18] G. Bossard and C. Ruef, Interacting non-BPS black holes, Gen. Rel. Grav. 44 (2012) 21 [arXiv: 1106.5806] [INSPIRE].

[19] O. Vasilakis and N.P. Warner, Mind the Gap: Supersymmetry Breaking in Scaling, Microstate Geometries, JHEP 10 (2011) 006 [arXiv:1104.2641] [InSPIRE].

[20] J. Polchinski and M.J. Strassler, The String dual of a confining four-dimensional gauge theory, hep-th/0003136 [INSPIRE].

[21] I.R. Klebanov and M.J. Strassler, Supergravity and a confining gauge theory: Duality cascades and chi SB resolution of naked singularities, JHEP 08 (2000) 052 [hep-th/0007191] [INSPIRE].

[22] H. Lin, O. Lunin and J.M. Maldacena, Bubbling AdS space and 1/2 BPS geometries, JHEP 10 (2004) 025 [hep-th/0409174] [INSPIRE].

[23] I. Bena and N.P. Warner, A Harmonic family of dielectric flow solutions with maximal supersymmetry, JHEP 12 (2004) 021 [hep-th/0406145] [INSPIRE].

[24] R. Penrose, Structure of space-time, in Battelle Rencontres: 1967 Lectures in Mathematics and Physics, C.M. DeWitt and J.A. Wheeler eds., Benjamin, New York, U.S.A. (1968), pg. 121-235.

[25] P.R. Brady and J.D. Smith, Black hole singularities: A Numerical approach, Phys. Rev. Lett. 75 (1995) 1256 [gr-qc/9506067] [INSPIRE].

[26] M. Dafermos, The Interior of charged black holes and the problem of uniqueness in general relativity, Commun. Pure Appl. Math. 58 (2005) 0445 [gr-qc/0307013] [INSPIRE]. 
[27] E. Poisson and W. Israel, Internal structure of black holes, Phys. Rev. D 41 (1990) 1796 [INSPIRE].

[28] D. Marolf, The dangers of extremes, Gen. Rel. Grav. 42 (2010) 2337 [arXiv:1005.2999] [INSPIRE].

[29] S.D. Mathur, What the information paradox is not, arXiv:1108.0302 [INSPIRE].

[30] A. Almheiri, D. Marolf, J. Polchinski and J. Sully, Black Holes: Complementarity or Firewalls?, arXiv:1207.3123 [INSPIRE].

[31] L. Susskind, Complementarity And Firewalls, arXiv:1207.4090.

[32] R. Bousso, Complementarity Is Not Enough, arXiv:1207.5192 [INSPIRE].

[33] Y. Nomura, J. Varela and S.J. Weinberg, Complementarity Endures: No Firewall for an Infalling Observer, arXiv:1207.6626 [INSPIRE].

[34] D. Harlow, Complementarity, not Firewalls, arXiv:1207.6243.

[35] S.D. Mathur and D. Turton, Comments on black holes I: The possibility of complementarity, arXiv:1208.2005 [INSPIRE].

[36] B.D. Chowdhury and A. Puhm, Is Alice burning or fuzzing?, arXiv:1208.2026 [INSPIRE].

[37] V. Jejjala, O. Madden, S.F. Ross and G. Titchener, Non-supersymmetric smooth geometries and D1-D5-P bound states, Phys. Rev. D 71 (2005) 124030 [hep-th/0504181] [INSPIRE].

[38] S. Giusto, S.F. Ross and A. Saxena, Non-supersymmetric microstates of the D1-D5-KK system, JHEP 12 (2007) 065 [arXiv:0708.3845] [INSPIRE].

[39] J.H. Al-Alawi and S.F. Ross, Spectral Flow of the Non-Supersymmetric Microstates of the D1-D5-KK System, JHEP 10 (2009) 082 [arXiv:0908.0417] [INSPIRE].

[40] I. Bena, S. Giusto, C. Ruef and N.P. Warner, A (Running) Bolt for New Reasons, JHEP 11 (2009) 089 [arXiv: 0909.2559] [INSPIRE].

[41] N. Bobev and C. Ruef, The Nuts and Bolts of Einstein-Maxwell Solutions, JHEP 01 (2010) 124 [arXiv:0912.0010] [INSPIRE].

[42] I. Bena, A. Puhm and B. Vercnocke, Metastable Supertubes and non-extremal Black Hole Microstates, JHEP 04 (2012) 100 [arXiv:1109.5180] [INSPIRE].

[43] S. Kachru, J. Pearson and H.L. Verlinde, Brane/flux annihilation and the string dual of a nonsupersymmetric field theory, JHEP 06 (2002) 021 [hep-th/0112197] [INSPIRE].

[44] B. Bates and F. Denef, Exact solutions for supersymmetric stationary black hole composites, JHEP 11 (2011) 127 [hep-th/0304094] [INSPIRE].

[45] F. Denef, Quantum quivers and Hall/hole halos, JHEP 10 (2002) 023 [hep-th/0206072] [INSPIRE].

[46] I. Bena, N. Bobev, C. Ruef and N.P. Warner, Supertubes in Bubbling Backgrounds: Born-Infeld Meets Supergravity, JHEP 07 (2009) 106 [arXiv:0812.2942] [INSPIRE].

[47] J. de Boer, S. El-Showk, I. Messamah and D. Van den Bleeken, Quantizing $N=2$ Multicenter Solutions, JHEP 05 (2009) 002 [arXiv:0807.4556] [INSPIRE].

[48] I. Bena, N. Bobev, C. Ruef and N.P. Warner, Entropy Enhancement and Black Hole Microstates, Phys. Rev. Lett. 105 (2010) 231301 [arXiv:0804.4487] [INSPIRE]. 
[49] S. Kachru et al., Towards inflation in string theory, JCAP 10 (2003) 013 [hep-th/0308055] [INSPIRE].

[50] I. Bena and N.P. Warner, One ring to rule them all... and in the darkness bind them?, Adv. Theor. Math. Phys. 9 (2005) 667 [hep-th/0408106] [INSPIRE].

[51] J.B. Gutowski and H.S. Reall, General supersymmetric AdS $S_{5}$ black holes, JHEP 04 (2004) 048 [hep-th/0401129] [INSPIRE].

[52] J.P. Gauntlett and J.B. Gutowski, General concentric black rings, Phys. Rev. D 71 (2005) 045002 [hep-th/0408122] [INSPIRE].

[53] H. Elvang, R. Emparan, D. Mateos and H.S. Reall, Supersymmetric black rings and three-charge supertubes, Phys. Rev. D 71 (2005) 024033 [hep-th/0408120] [INSPIRE].

[54] M. Cvetič and D. Youm, General rotating five-dimensional black holes of toroidally compactified heterotic string, Nucl. Phys. B 476 (1996) 118 [hep-th/9603100] [inSPIRE].

[55] I. Bena, G. Giecold, M. Graña and N. Halmagyi, On The Inflaton Potential From Antibranes in Warped Throats, JHEP 07 (2012) 140 [arXiv:1011.2626] [INSPIRE].

[56] A. Dymarsky, On gravity dual of a metastable vacuum in Klebanov-Strassler theory, JHEP 05 (2011) 053 [arXiv: 1102.1734] [INSPIRE].

[57] I. Bena, G. Giecold, M. Graña, N. Halmagyi and S. Massai, On Metastable Vacua and the Warped Deformed Conifold: Analytic Results, arXiv:1102.2403 [INSPIRE].

[58] I. Bena, G. Giecold, M. Graña, N. Halmagyi and S. Massai, The backreaction of anti-D3 branes on the Klebanov-Strassler geometry, arXiv:1106.6165 [INSPIRE].

[59] S.D. Mathur, Black Holes and Beyond, Annals Phys. 327 (2012) 2760 [arXiv:1205.0776] [INSPIRE].

[60] S.D. Mathur, Black holes and holography, arXiv:1207.5431 [INSPIRE].

[61] M. Van Raamsdonk, Comments on quantum gravity and entanglement, arXiv:0907.2939 [INSPIRE].

[62] M. Van Raamsdonk, Building up spacetime with quantum entanglement, Gen. Rel. Grav. 42 (2010) 2323 [arXiv: 1005.3035] [InSPIRE].

[63] D. Anninos, T. Anous, F. Denef, G. Konstantinidis and E. Shaghoulian, Supergoop Dynamics, arXiv: 1205.1060 [INSPIRE].

[64] J. Preskill, P. Schwarz, A.D. Shapere, S. Trivedi and F. Wilczek, Limitations on the statistical description of black holes, Mod. Phys. Lett. A 6 (1991) 2353 [InSPIRE].

[65] P. Kraus and F. Wilczek, Effect of selfinteraction on charged black hole radiance, Nucl. Phys. B 437 (1995) 231 [hep-th/9411219] [INSPIRE].

[66] V. Cardoso, O.J. Dias, J.L. Hovdebo and R.C. Myers, Instability of non-supersymmetric smooth geometries, Phys. Rev. D 73 (2006) 064031 [hep-th/0512277] [INSPIRE].

[67] S.G. Avery, B.D. Chowdhury and S.D. Mathur, Emission from the D1D5 CFT, JHEP 10 (2009) 065 [arXiv:0906.2015] [INSPIRE].

[68] I. Bena, M. Graña and N. Halmagyi, On the Existence of Meta-stable Vacua in Klebanov-Strassler, JHEP 09 (2010) 087 [arXiv:0912.3519] [INSPIRE].

[69] I. Bena, G. Giecold and N. Halmagyi, The Backreaction of Anti-M2 Branes on a Warped Stenzel Space, JHEP 04 (2011) 120 [arXiv:1011.2195] [InSPIRE]. 
[70] G. Giecold, E. Goi and F. Orsi, Assessing a candidate IIA dual to metastable supersymmetry-breaking, JHEP 02 (2012) 019 [arXiv:1108.1789] [INSPIRE].

[71] S. Massai, A Comment on anti-brane singularities in warped throats, arXiv:1202.3789 [INSPIRE].

[72] J. Blaback et al., The problematic backreaction of SUSY-breaking branes, JHEP 08 (2011) 105 [arXiv: 1105.4879] [INSPIRE].

[73] I. Bena, M. Graña, S. Kuperstein and S. Massai, Anti-D3's - Singular to the Bitter End, arXiv:1206.6369 [INSPIRE].

[74] S.D. Mathur, Tunneling into fuzzball states, Gen. Rel. Grav. 42 (2010) 113 [arXiv:0805.3716] [INSPIRE].

[75] B.D. Chowdhury and S.D. Mathur, Fractional Brane State in the Early Universe, Class. Quant. Grav. 24 (2007) 2689 [hep-th/0611330] [INSPIRE].

[76] S.D. Mathur, What can the information paradox tell us about the early Universe?, arXiv: 1205.3140 [INSPIRE].

[77] A. Saxena, G. Potvin, S. Giusto and A.W. Peet, Smooth geometries with four charges in four dimensions, JHEP 04 (2006) 010 [hep-th/0509214] [INSPIRE].

[78] F. Denef, Supergravity flows and D-brane stability, JHEP 08 (2000) 050 [hep-th/0005049] [INSPIRE].

[79] B.D. Chowdhury and B. Vercnocke, New instability of non-extremal black holes: spitting out supertubes, JHEP 02 (2012) 116 [arXiv:1110.5641] [INSPIRE]. 